\title{
Characterization of tie-breaking plurality rules
}

\author{
Hiroki Saitoh ${ }^{1} \mathbb{D}$
}

Received: 13 April 2021 / Accepted: 2 December 2021 / Published online: 30 January 2022

() The Author(s) 2021

\begin{abstract}
We introduce a new condition for social choice functions, called "equal treatment of congruent distributions." It requires some invariance between two preference profiles that share a type of congruity property with respect to the associated distributions of votes. It also implies two equal treatment conditions: one is a natural weakening of anonymity, which is the most standard equal treatment condition for individuals, and the other is a natural weakening of neutrality, which is the most standard equal treatment one for alternatives. Thus, equal treatment of congruent distributions plays the role of weak equal treatment conditions both for individuals and for alternatives. As our main results, we characterize a class of social choice functions that satisfy equal treatment of congruent distributions and some mild positive responsiveness conditions, and it is shown to coincide with the class of tie-breaking plurality rules, which are selections of the well-known plurality rule.
\end{abstract}

\section{Introduction}

We consider a collective choice problem in which a society chooses from the set of feasible alternatives on the basis of individual preferences. The society attempts to make decisions not only by treating individuals as equally as possible, but also by treating alternatives as equally as possible. In other words, it respects equal treatment (or symmetry) criteria. The numbers of alternatives and individuals are assumed to be both fixed. The preferences of individuals are linear orders over the set of alternatives. We particularly focus on the procedures of outputting alternatives as social outcomes (i.e., social choice correspondences), not on the ones of outputting social rankings of alternatives (i.e., social preference correspondences). A social choice correspondence is a procedure that assigns a nonempty subset of the set of alternatives to every possible profile of individual preferences called a preference profile. One of the most well-known social choice correspondences is the

Hiroki Saitoh

hsaitoh@eco.meijigakuin.ac.jp

1 Faculty of Economics, Meiji Gakuin University, 1-2-37, Shirokanedai, Minato-ku,

Tokyo 108-8636, Japan 
plurality rule, in which individuals vote for their most preferred alternatives. Then, the rule chooses alternatives having the maximal votes, ${ }^{1}$

Although the analyses of social choice correspondences, including those about the plurality rule, have been extensively conducted thus far, they cannot be applied to the most essential situation in which only one alternative must be chosen since such procedures may output two or more alternatives (i.e., there is a possibility of a tie) for a preference profile. Situations requiring choosing a single alternative occur universally, and there is no doubt about the importance of dealing with such situations. To accommodate these, it is appropriate to adopt social choice functions ${ }^{2}$ which assign a single alternative to every possible preference profile. However, the serious conflict described below between two major equal treatment conditions (i.e., anonymity and neutrality) make the analysis of social choice functions difficult from the perspective ${ }^{3}$ of equal treatment criteria. In this paper, we restrict our attention to social choice functions and address the critical problem of the impossibility of achieving full equal treatment resulting from the conflict, with the aiming of achieving some equal treatment.

Anonymity and neutrality are the most standard equal treatment conditions. Anonymity, which is a condition for the equal treatment of individuals, requires that a social choice correspondence should be independent of individual names. On the other hand, neutrality, which is a condition for the equal treatment of alternatives, requires that it should be independent of the names of the alternatives. Most studies rely on these conditions as basic axioms for characterizations ${ }^{4}$ of various social choice correspondences, including the plurality rule.

Although anonymity and neutrality play important roles for characterization of social choice correspondences, it is well-known that they are generally incompatible $^{5}$ on social choice functions. In other words, they are compatible only under a specific condition on the numbers of alternatives and individuals. Concretely, Moulin (1983) showed that a necessary and sufficient condition for the existence of a social choice function that satisfies both anonymity and neutrality is that the number of alternatives cannot be written as the sum of some divisors (other than 1) of the number of individuals. Therefore, given the impossibility result, if we attempt to pursue social choice functions from the perspective of

\footnotetext{
1 This rule is one of the scoring rules which include the well-known Borda rule. For the details of scoring rules, see Young (1975).

2 Some studies treat the definition of a social choice function as one of axioms on social choice correspondences. Such axiom is often referred to as resoluteness.

3 Social choice functions also suffer from the perspective of strategic behaviors, as examined by the important works of Gibbard (1973) and Satterthwaite (1975), which provided the Gibbard-Satterthwaite theorem. Examples of studies on social choice functions in collective choice problems from the aspects of strategic issues include those of Pazner and Wesley (1978), Peleg (1978), Moulin (1979, 1980), Sanver (2009), and Campbell and Kelly (2016).

${ }^{4}$ Examples, restricting our attention to characterizations of the plurality rule and its related rules, include those of Richelson (1978), Ching (1996), Baharad and Nitzan (2005), Ju (2005), Yeh (2008), Sekiguchi (2012), Kelly and Qi (2016), and Öztürk (2020).

5 For the analysis of the incompatibility of anonymity and neutrality, see Moulin (1983), Bubboloni and Gori (2014), Campbell and Kelly (2015), Doğan and Giritligil (2015), and Ozkes and Sanver (2021).
} 
equal treatment criteria, then we must generally give up either anonymity, neutrality, or both. In related research on these lines, Fishburn and Gehrlein (1977) and Nitzan and Paroush (1981) analyzed binary social choice functions satisfying neutrality without imposing anonymity in a binary decision problem, which is a special case of our setting. Campbell and Kelly (2013) and Jeong and Ju (2017) also treated the binary decision problem. Both studies analyzed binary social choice functions satisfying anonymity and some weakenings of neutrality. Recently, Bubboloni and Gori (2021) and Ozkes and Sanver (2021) treated the same setting as ours. Bubboloni and Gori (2021) employed two axioms: one is a weakening of anonymity and the other is a weakening of neutrality. They gave a condition for which those two axioms are compatible. Ozkes and Sanver (2021) analyzed social choice functions satisfying anonymity and a weakening of neutrality. They investigated the existence problem of social choice functions satisfying those conditions.

In this paper, we adopt the third option described above to pursue social choice functions from the perspective of equal treatment criteria. That is, we impose neither anonymity nor neutrality. Instead, we introduce a new condition called equal treatment of congruent distributions (ETCD), which is linked to some equal treatment conditions both for individuals and for alternatives, as described below.

To explain ETCD, suppose, as with the plurality rule, that individuals vote for their most preferred alternatives according to their preferences. We then focus on the associated distribution of votes under a preference profile. If two given preference profiles share the same shape of the distribution of votes except for the names of alternatives and individuals, then we say that these preference profiles have congruent distributions. ETCD requires that under two preference profiles having congruent distributions, the chosen two alternatives should share the same number of votes. As a basic property, ETCD implies nondictatorship whenever the number of individuals is three or more (Fact 1 in Sect. 3.1).

ETCD can be regarded as linked to some equal treatment conditions from two perspectives. The first perspective is related to the situations in which anonymity and neutrality are compatible. Under such situations, it is shown that ETCD is equivalent to anonymity and neutrality whenever the number of alternatives is two (Proposition 1 in Sect. 3.2). Furthermore, it is shown that ETCD is a conditional generalization of anonymity and neutrality whenever the number of alternatives is more than two (Proposition 2 in Sect. 3.2). The second perspective is related to the situations in which anonymity and neutrality are incompatible. Even in such situations, ETCD is available, and it is shown that it implies two equal treatment conditions: anonymity in the number of votes (ANV) and neutrality in the number of votes (NNV) (Proposition 3 in Sect. 3.2). ANV, which is weaker than anonymity, requires that the number of votes for the chosen alternatives should be independent of the names of individuals. On the other hand, NNV, which is weaker than neutrality, requires that the number of votes for the chosen alternatives should be independent of the names of alternatives. ANV and NNV can be interpreted as weak equal treatment conditions for individuals and for alternatives, respectively. These requirements are mild, but natural and intuitive. It is easily checked that ETCD implies both ANV and NNV. Thus, even in the situations such that anonymity and neutrality are incompatible, 
ETCD can play the role of weak equal treatment conditions both for individuals and for alternatives.

Using ETCD, we characterize a class of social choice functions called the class of tie-breaking plurality (TBP) rules, which are social choice functions derived from the plurality rule in a natural way. Specifically, a TBP rule always selects a single alternative among the alternatives having the maximal votes (i.e., it breaks a tie in some manner if there are two or more such alternatives). Formally, a TBP rule is identified with a selection of the plurality rule, ${ }^{6}$

As the main results of the paper, we give two characterizations of the class of TBP rules by ETCD and two types of positive responsiveness conditions called monotonicity and weak monotonicity. Monotonicity requires that if an alternative chosen at a given preference profile becomes most preferred whereas the relative rank of other alternatives remains the same under a new preference of an individual, then the alternative should remain chosen at the new preference profile. On the other hand, weak monotonicity ${ }^{7}$ requires that if an alternative chosen at a given preference profile becomes from second preferred to most preferred, whereas the relative rank of other alternatives remains the same under a new preference of an individual, then the alternative should remain chosen by the new preference profile. Although there are various types of definitions of monotonicity, our monotonicity conditions are mild requirements in the sense that they focus only on an improvement of the rank of an alternative to the top rank. Especially, weak monotonicity is a very weak condition. Our first result (Theorem 1 in Sect. 5) states that the class of TBP rules is characterized by ETCD and monotonicity. Our second result (Theorem 2 in Sect. 5) states that if the number of individuals is sufficiently large, then the class of TBP rules is characterized by ETCD and weak monotonicity.

Our research is significant in two ways. The first regards the proposal of a new easy-to-use condition (i.e., ETCD), which implies some natural equal treatment conditions both for individuals and for alternatives. The definition of ETCD is simple, intuitive, and easy to understand. ETCD focuses on the distribution of votes, which is one of the easiest data to handle in a preference profile. It is available without any essential restriction on the numbers of alternatives and individuals. In existing research that tried to avoid conflicts between equal treatments of individuals vs. alternatives, some weakenings of anonymity and/or neutrality were employed. Although it is usually necessary to pay attention to the possibilities of further incompatibilities between such conditions, ETCD can be used without worrying about them. Furthermore, even if a society takes a great interest in treating individuals (resp. alternatives) more equally, it is also possible to make ETCD and anonymity (resp. neutrality) compatible. The second is the characterizations of a natural class of social choice functions (i.e., TBP rules). TBP rules are also simple, intuitive, and easy to understand. Our results support TBP rules from equal treatment criteria and

\footnotetext{
${ }^{6}$ Another type of well-known social choice function is a run-off voting rule which is a method of electing a single alternative by continuing to remove alternatives in a certain manner via multiple rounds until only one alternative remains. Since the chosen alternative at the final round is not always the one with the maximal votes in the first round, a run-off voting rule is generally different from a TBP rule.

7 This condition is due to Kelly and Qi’s (2016) monotonicity.
} 
positive responsiveness criteria. Apart from those, it is also argued that TBP rules are supported by efficiency criteria and that they are partially supported by incentive compatibility criteria.

The rest of the paper is organized as follows: Sect. 2 gives definitions and notations and reviews the plurality rule. Section 3 describes ETCD. Section 4 defines TBP rules. Section 5 states our characterization results for TBP rules. Section 6 discusses TBP rules and states some open questions. Appendix A shows the independence of axioms. Appendices B and $\mathrm{C}$ are devoted to the proofs of our results.

\section{Definition}

Let $N=\{1, \ldots, n\}$ be the set of individuals with $n \geq 3$. Let $X$ be the set of feasible alternatives with $|X| \equiv m \geq 2 .{ }^{8}$ Each individual $i \in N$ has a preference $P_{i}$, which is a complete, transitive, and anti-symmetric binary relation on $X$. Let $\mathscr{P}$ be the set of all preferences on $X$. A preference profile, or simply, a profile $P=\left(P_{i}\right)_{i \in N}$ is an element of $\mathscr{P}^{N}$. A social choice correspondence $F$ is a mapping from $\mathscr{P}^{N}$ to $2^{X} \backslash\{\emptyset\}$. If a social choice correspondence $F$ satisfies that for each $P \in \mathscr{P}^{N},|F(P)|=1$ (i.e., it always selects a single alternative), we say that $F$ is a social choice function. To distinguish functions from correspondences, we employ the lower case $f$ as the generic notation for social choice functions. For abbreviation, we write $f(P)=x$ instead of $f(P)=\{x\}$.

A well-known social choice correspondence is the plurality rule. To introduce the rule, we provide some notations. Given $i \in N$ and $P_{i} \in \mathscr{P}$, let $t\left(P_{i}\right) \in X$ be such that for each $x \in X \backslash\left\{t\left(P_{i}\right)\right\}, t\left(P_{i}\right) P_{i} x$ (i.e., it is the most preferred, or equivalently, top-ranked, alternative under $\left.P_{i}\right)$. Given $x \in X$ and $P=\left(P_{i}\right)_{i \in N} \in \mathscr{P}^{N}$, let $N(x, P)=\left\{i \in N: t\left(P_{i}\right)=x\right\}$, which is the set of individuals whose top-ranked alternatives are $x$ under $P$. Then, although $|N(x, P)|$ indicates the number of individuals whose top-ranked alternatives are $x$ under $P$, it is identified with the number of votes for $x$ under $P$ in the situation where individuals vote for their most preferred alternatives according to their preferences. The plurality rule is then defined as follows.

The plurality rule $F_{p}$ is a social choice correspondence such that for each $P \in \mathscr{P}^{N}$,

$$
F_{p}(P)=\{x \in X:|N(x, P)| \geq|N(y, P)| \text { for all } y \in X\} .
$$

That is, it always chooses alternatives having the maximal votes. It is known that the plurality rule satisfies many properties. The most standard ones are anonymity and neutrality.

Anonymity: For each $P=\left(P_{i}\right)_{i \in N} \in \mathscr{P}^{N}$ and each $P^{\prime}=\left(P_{i}^{\prime}\right)_{i \in N} \in \mathscr{P}^{N}$, if there exists a bijection $\pi: N \rightarrow N$ such that for each $j \in N, P_{\pi(j)}^{\prime}=P_{j}$, then $F(P)=F\left(P^{\prime}\right)$.

\footnotetext{
${ }^{8}$ Given a set $A,|A|$ indicates the cardinality of $A$. All statements and examples in this paper apply to all $m \geq 2$ and $n \geq 3$, unless otherwise noted.
} 
Neutrality: For each $P=\left(P_{i}\right)_{i \in N} \in \mathscr{P}^{N}$ and each $P^{\prime}=\left(P_{i}^{\prime}\right)_{i \in N} \in \mathscr{P}^{N}$, if there exists a bijection $\sigma: X \rightarrow X$ such that for each $j \in N$ and each $x, y \in X, x P_{j} y$ if and only if $\sigma(x) P_{j}^{\prime} \sigma(y)$, then $\sigma(F(P))=F\left(P^{\prime}\right)$.

Anonymity and neutrality are equal treatment, or symmetry, conditions on individuals and alternatives, respectively. Anonymity requires that a social choice correspondence be independent on the names of individuals. Neutrality requires that it should be independent on the names of alternatives.

Furthermore, the plurality rule satisfies the following three properties:

Tops-only: For each $P=\left(P_{i}\right)_{i \in N} \in \mathscr{P}^{N}$ and each $P^{\prime}=\left(P_{i}^{\prime}\right)_{i \in N} \in \mathscr{P}^{N}$, if for all $j \in N, t\left(P_{j}\right)=t\left(P_{j}^{\prime}\right)$, then $F(P)=F\left(P^{\prime}\right)$.

Tops-only is a simplicity condition in which a social choice correspondence depends only on the list of top-ranked alternatives of individuals. Especially in decision making in a large society, some simplification of the rules is important for smooth execution of decision making. Note that this condition is automatically satisfied by all social choice correspondences whenever $m=2$.

Given $i \in N, P_{i} \in \mathscr{P}$, and $x \in X, \tilde{P}\left(P_{i} ; x\right) \in \mathscr{P}$ is defined by $t\left(\tilde{P}\left(P_{i} ; x\right)\right)=x$ and for each $y, z \in X \backslash\{x\}, y \tilde{P}\left(P_{i} ; x\right) z$ if and only if $y P_{i} z$. That is, $\tilde{P}\left(P_{i} ; x\right)$ is a transformation of $P_{i}$ obtained by moving $x$ to the top rank and by preserving the relative rank of alternatives other than $x$. Given $P=\left(P_{i}\right)_{i \in N} \in \mathscr{P}^{N}, j \in N$, and $P_{j}^{\prime} \in \mathscr{P}$, we write a profile $\left(P_{j}^{\prime},\left(P_{i}\right)_{i \neq j}\right) \in \mathscr{P}^{N}$ as $\left(P_{j}^{\prime}, P_{-j}\right)$.

Monotonicity: ${ }^{9}$ For each $P=\left(P_{i}\right)_{i \in N} \in \mathscr{P}^{N}$, each $j \in N$, and each $x \in X$, if $x \in F(P)$, then $x \in F\left(\tilde{P}\left(P_{j} ; x\right), P_{-j}\right)$ and $F\left(\tilde{P}\left(P_{j} ; x\right), P_{-j}\right) \subseteq F(P)$.

Monotonicity is a positive responsiveness condition in which, if an alternative chosen at a given profile is moved to the top rank under a new preference of an individual, then the alternative remains chosen under the new profile, and the set of chosen alternatives does not expand.

Given $i \in N$ and $P_{i} \in \mathscr{P}$, let $t_{2}\left(P_{i}\right) \in X$ be such that $t_{2}\left(P_{i}\right) \neq t\left(P_{i}\right)$ and for each $x \in X \backslash\left\{t\left(P_{i}\right), t_{2}\left(P_{i}\right)\right\}$ (if such $x$ exists), $t_{2}\left(P_{i}\right) P_{i} x$, i.e., it is the second-ranked alternative under $P_{i}$.

Weak Monotonicity: ${ }^{10}$ For each $P=\left(P_{i}\right)_{i \in N} \in \mathscr{P}^{N}$, each $j \in N$, and each $x \in X$, if $x \in F(P)$ and $x=t_{2}\left(P_{j}\right)$, then $x \in F\left(\tilde{P}\left(P_{j} ; x\right), P_{-j}\right)$ and $F\left(\tilde{P}\left(P_{j} ; x\right), P_{-j}\right) \subseteq F(P)$.

That is, weak monotonicity means that, if an alternative chosen at a profile is changed from the second rank to the top rank under a new preference of an individual, then the alternative remains at the new profile chosen, and the set of chosen

\footnotetext{
9 Öztürk (2020) employed a monotonicity condition that is different from ours. Although these are independent under social choice correspondences, Öztürk's (2020) monotonicity is stronger than ours under social choice functions.

10 This condition is a weaker version of Kelly and Qi's (2016) monotonicity. Their monotonicity requires that, under the same assumption, $x$ should be uniquely chosen at the new profile. Note that weak monotonicity becomes equivalent to their monotonicity under social choice functions.
} 
alternatives does not expand. It is obvious that weak monotonicity coincides with monotonicity whenever $m=2$. On the other hand, it is implied by monotonicity whenever $m \geq 3$.

As mentioned, we focus on social choice functions. Thus, it is useful to rewrite the definitions of the above two monotonicity conditions for social choice functions.

Monotonicity (for social choice functions): For each $P=\left(P_{i}\right)_{i \in N} \in \mathscr{P}^{N}$, each $j \in N$, and each $x \in X$, if $x=f(P)$, then $x=f\left(\tilde{P}\left(P_{j} ; x\right), P_{-j}\right)$.

Weak Monotonicity (for social choice functions): For each $P=\left(P_{i}\right)_{i \in N} \in \mathscr{P}^{N}$, each $j \in N$, and each $x \in X$, if $x=f(P)$ and $x=t_{2}\left(P_{j}\right)$, then $x=f\left(\tilde{P}\left(P_{j} ; x\right), P_{-j}\right)$.

\section{Equal treatment of congruent distributions}

\subsection{Definition}

As mentioned in Sect. 1, social choice functions suffer from the conflict of anonymity and neutrality, meaning that we must generally give up either anonymity, neutrality, or both. In this paper, we take the third option, and so we employ neither anonymity nor neutrality. Instead, we introduce a new condition that plays the role of some equal treatment conditions both for individuals and for alternatives. We call it equal treatment of congruent distributions (ETCD).

ETCD requires that, if two given preference profiles share some kind of congruity property with respect to the associated distributions of votes, the chosen alternatives at those profiles should share the same number of votes.

For an intuitive explanation of ETCD prior to its formal definition, consider the mayoral election of a town, in which 10,000 townspeople (i.e., individuals) vote for their top-ranked candidates (i.e., alternatives) among six candidates. Then, consider the following two distributions of votes that are derived from profiles $P$ and $P^{\prime}$, respectively:

The distribution of votes under $P:\left\{\begin{array}{l}\left|N\left(x_{1}, P\right)\right|=\left|N\left(x_{2}, P\right)\right|=\left|N\left(x_{3}, P\right)\right|=3,333, \\ \left|N\left(x_{4}, P\right)\right|=1, \text { and } \\ \left|N\left(x_{5}, P\right)\right|=\left|N\left(x_{6}, P\right)\right|=0 .\end{array}\right.$

The distribution of votes under $P^{\prime}:\left\{\begin{array}{l}\left|N\left(x_{1}, P^{\prime}\right)\right|=\left|N\left(x_{4}, P^{\prime}\right)\right|=\left|N\left(x_{6}, P^{\prime}\right)\right|=3,333, \\ \left|N\left(x_{2}, P^{\prime}\right)\right|=1, \text { and } \\ \left|N\left(x_{3}, P^{\prime}\right)\right|=\left|N\left(x_{5}, P^{\prime}\right)\right|=0 .\end{array}\right.$

Recall that $\left|N\left(x_{k}, P\right)\right|$ is identified with the number of votes for $x_{k}$ under $P$. Then, a list $\left(\left|N\left(x_{k}, P\right)\right|\right)_{k=1, \ldots, 6}$ forms the distribution of votes under $P$. Note that there is a clear similarity between $P$ and $P^{\prime}$. That is, except for the names of alternatives and individuals, these profiles exhibit the same shape of vote distribution. Concretely, for both profiles, three alternatives have 3,333 votes, one alternative has one, and two alternatives have none. We say that two profiles exhibiting the same shape of the distribution of votes have congruent distributions. 
Under the incompatibility of anonymity and neutrality, if a society tries to make a decision that is based on equal treatment criteria for individuals and alternatives, what condition should be adopted for a social choice function? For example, in the above example, it seems that a social choice function choosing an alternative that has 3,333 votes for $P$, and choosing an alternative that has one or no vote for $P^{\prime}$ causes an apparent asymmetry. In other words, it seems that some of alternatives and/or individuals are treated quite asymmetrically. Here, to reduce the asymmetry between two profiles having congruent distributions, we adopt the condition as an equal treatment requirement that the chosen alternatives at those profiles always should share the same number of votes. This is ETCD. For the above example, if a social choice function $f$ satisfies ETCD, then $f(P) \in\left\{x_{1}, x_{2}, x_{3}\right\}$ if and only if $f\left(P^{\prime}\right) \in\left\{x_{1}, x_{4}, x_{6}\right\}, f(P)=x_{4}$ if and only if $f\left(P^{\prime}\right)=x_{2}$, and $f(P) \in\left\{x_{5}, x_{6}\right\}$ if and only if $f\left(P^{\prime}\right) \in\left\{x_{3}, x_{5}\right\}$.

ETCD requires an invariance up to choosing from among alternatives having the same number of votes for both two profiles having congruent distributions. This means that it does not require anything about which alternative should be chosen from among such alternatives for each of those profiles. Thus, even if $P$ and $P^{\prime}$ satisfy the assumptions both of anonymity and neutrality in the above example, a further asymmetry between the result of a choice from $\left\{x_{1}, x_{2}, x_{3}\right\}$ under $P$ and that of a choice from $\left\{x_{1}, x_{4}, x_{6}\right\}$ under $P^{\prime}$ may also occur. However, since it is generally caused by the incompatibility of anonymity and neutrality, such asymmetry is inevitable as long as we employ social choice functions.

To define ETCD formally, we define a notation. For given $P \in \mathscr{P}^{N}$ and given $\ell \in\{0,1, \ldots, n\}$, let $X_{\ell}(P)=\{x \in X:|N(x, P)|=\ell\} . X_{\ell}(P)$ is the set of alternatives that get $\ell$ vote(s) under $P$. Note that $\bigcup_{\ell \geq 1} X_{\ell}(P)\left(=X \backslash X_{0}(P)\right)$ forms the set of alternatives that are most preferred by some individuals under $P$ (i.e., it is the set of top-ranked alternatives under $P$ ).

For given $P \in \mathscr{P}^{N}$ and $P^{\prime} \in \mathscr{P}^{N}$, we say that $P$ and $P^{\prime}$ have congruent distributions $(\mathrm{CD})$ if for all $\ell \in\{0,1, \ldots, n\},\left|X_{\ell}(P)\right|=\left|X_{\ell}\left(P^{\prime}\right)\right|$. That is, two profiles having $\mathrm{CD}$ exhibit the same shape of the distribution of votes, except for the names of alternatives and individuals. ETCD is then defined as follows.

Equal Treatment of Congruent Distributions (ETCD): For each $P \in \mathscr{P}^{N}$ and each $P^{\prime} \in \mathscr{P}^{N}$, if $P$ and $P^{\prime}$ have congruent distributions, then for all $\ell \in\{0,1, \ldots, n\}, f(P) \in X_{\ell}(P)$ if and only if $f\left(P^{\prime}\right) \in X_{\ell}\left(P^{\prime}\right)$.

This requires that the chosen alternatives under two given profiles having CD should share the same number of votes. Note that although ETCD focuses on the distribution of votes, it is distinctly different from tops-only. In other words, under ETCD, for two profiles with the same list of top-ranked alternatives of individuals, the social choice function may assign different alternatives to each other. Thus, a social choice function that satisfies ETCD generally uses more information about a preference profile than a social choice function that satisfies topsonly. The following fact states that ETCD implies nondictatorship. 
Fact 1 If a social choice function $f$ satisfies equal treatment of congruent distributions, then it is nondictatorial in the sense that there is no dictator $j_{0} \in N$ such that for all $P=\left(P_{i}\right)_{i \in N} \in \mathscr{P}^{N}, f(P)=t\left(P_{j_{0}}\right)$.

Proof of Fact 1: Suppose the contrary that $f$ satisfies equal treatment of congruent distributions and that it is dictatorial. Let $j_{0} \in N$ be a dictator. Let $P=\left(P_{i}\right)_{i \in N} \in \mathscr{P}^{N}$ and $P^{\prime}=\left(P_{i}^{\prime}\right)_{i \in N} \in \mathscr{P}^{N}$ be such that (i) $t\left(P_{j_{0}}\right)=x$ and for all $j \neq j_{0}, t\left(P_{j}\right)=y$ with $y \neq x$ and (ii) $t\left(P_{j^{\prime}}^{\prime}\right)=y$ with $j^{\prime} \neq j_{0}$ and for all $j \neq j^{\prime}, t\left(P_{j}^{\prime}\right)=x$. It then holds that $f(P)=f\left(P^{\prime}\right)=x$. It is obvious that $P$ and $P^{\prime}$ have CD. However, we have $f(P)=x \in X_{1}(P)$ and $f\left(P^{\prime}\right)=x \in X_{n-1}\left(P^{\prime}\right)$, a contradiction to ETCD since $n \geq 3$.

\subsection{Relationship to other axioms}

ETCD has a relationship with some equal treatment conditions. At first, under a pair of $m$ and $n$ such that anonymity and neutrality are compatible, ETCD is equivalent to anonymity and neutrality whenever $m=2$. Furthermore, it is a conditional generalization of anonymity and neutrality whenever $m \geq 3$. To see this, we first give a condition for the compatibility of anonymity and neutrality, as obtained by Moulin (1983). Let $(m, n)$ denote a tuple of the number of alternatives and the number of individuals.

Fact 2 (Moulin (1983)) A necessary and sufficient condition on ( $m, n)$ for the existence of a social choice function that satisfies both anonymity and neutrality is that $m$ cannot be written as the sum of some divisors (other than 1) of $n$.

Note that $(2, n)$ satisfies the condition of Fact 2 if and only if $n$ is odd. By this fact, we have the following equivalence result.

Proposition 1 Assume that $m=2$ and $n$ is odd. Then, a social choice function $f$ satisfies anonymity and neutrality if and only if it satisfies equal treatment of congruent distributions.

The proof is given in Appendix B.1. Note that anonymity (resp. neutrality) itself does not imply ETCD (Example 6 (resp. 7) in Appendix A.1).

For $(m, n)$ with $m \geq 3$, even if it satisfies the condition of Fact 2 , the similar equivalence does not hold. That is, there exists a social choice function that satisfies ETCD, but violates both anonymity and neutrality (Example 5 in Appendix A.1). Conversely, the Coombs social choice function $^{11}$ due to Moulin (1983) satisfies anonymity and neutrality, but violates ETCD since the shape of the distribution of votes does not matter under the social choice function. However, under tops-only,

\footnotetext{
11 This social choice function is well-defined under $(m, n)$ such that every prime factor of $n$ exceeds $m$. Note that this condition implies the condition of Fact 2. Similar to run-off voting rules, it continues to eliminate alternatives until only one alternative remains. For the elimination method of the Coombs social choice function, see Example 3 in Sect. 4.
} 
anonymity and neutrality imply ETCD for any $(m, n)$ such that anonymity and neutrality are compatible.

Proposition 2 Assume that $(m, n)$ with $m \geq 3$ is such that anonymity and neutrality are compatible. Then, if a social choice function $f$ satisfies anonymity, neutrality, and tops-only, then it satisfies equal treatment of congruent distributions.

The proof is given in Appendix B.2. Note that the Coombs social choice function violates tops-only since non-top-ranked alternatives matter under the social choice function. Proposition 2 means that under tops-only, ETCD is a generalization of anonymity and neutrality whenever $m \geq 3$.

Next, consider any pair $(m, n)$ such that anonymity and neutrality may be incompatible. ETCD then implies the following two conditions that can be considered as equal treatment conditions for individuals and for alternatives: anonymity in the number of votes and neutrality in the number of votes, respectively.

Anonymity in the Number of Votes (ANV): For each $P=\left(P_{i}\right)_{i \in N} \in \mathscr{P}^{N}$ and each $P^{\prime}=\left(P_{i}^{\prime}\right)_{i \in N} \in \mathscr{P}^{N}$, if there exists a bijection $\pi: N \rightarrow N$ such that for each $j \in N$, $P_{\pi(j)}^{\prime}=P_{j}$, then for all $\ell \in\{0,1, \ldots, n\}, f(P) \in X_{\ell}(P)$ if and only if $f\left(P^{\prime}\right) \in X_{\ell}\left(P^{\prime}\right)$.

Neutrality in the Number of Votes (NNV): For each $P=\left(P_{i}\right)_{i \in N} \in \mathscr{P}^{N}$ and each $P^{\prime}=\left(P_{i}^{\prime}\right)_{i \in N} \in \mathscr{P}^{N}$, if there exists a bijection $\sigma: X \rightarrow X$ such that for each $j \in N$ and each $x, y \in X, x P_{j} y$ if and only if $\sigma(x) P_{j}^{\prime} \sigma(y)$, then for all $\ell \in\{0,1, \ldots, n\}$, $f(P) \in X_{\ell}(P)$ if and only if $f\left(P^{\prime}\right) \in X_{\ell}\left(P^{\prime}\right)$.

ANV requires that the number of votes for the chosen alternatives should be independent of the names of individuals. NNV requires that the number of votes for the chosen alternatives should be independent of the names of alternatives. The following proposition, which summarizes the basic logical relationships, justifies these two conditions to be equal treatment conditions.

Proposition 3 The following holds:

[1] If a social choice function $f$ satisfies anonymity, then it satisfies anonymity in the number of votes. Moreover, for $(m, n)$ such that $m=2$ and $n$ is odd, the converse is also true.

[2] If a social choice function f satisfies neutrality, then it satisfies neutrality in the number of votes. Moreover, for $(m, n)$ such that $m=2$ and $n$ is odd, the converse is also true.

[3] If a social choice function $f$ satisfies equal treatment of congruent distributions, then it satisfies both anonymity in the number of votes and neutrality in the number of votes. Moreover, if $m=2$, then the converse is also true.

The first part of every statement follows immediately from the definitions. The proof of the second part of every statement is given in Appendix B.3. For [1] and [2], except for $(m, n)$ such that $m=2$ and $n$ is odd, the converse is not true. For 
example, $f_{5}$ in Example 5 of Appendix A.1 satisfies ETCD, and hence by [3], does ANV and NNV, but violates anonymity and neutrality. Note that for the second part of [3], the condition on $n$ is not necessary. Also, if $m \geq 3$, then the converse is not true. The Coombs social choice function, which satisfies ANV and NNV, but violates ETCD, is an example.

By [1] (resp. [2]), ANV (resp. NNV) can be interpreted as an equal treatment condition for individuals (resp. for alternatives), which is weaker than anonymity (resp. neutrality). ANV and NNV are both mild requirements for equal treatment, but they are natural and intuitive. By [3], ETCD is a condition that implies ANV and NNV. This means that ETCD plays the role of weak equal treatment conditions both for individuals and for alternatives. Furthermore, it is effective even under situations where anonymity and neutrality are incompatible, that is, situations in which they cannot be relied upon.

Note that tops-only also shares the similar invariance property of ANV and NNV. That is, if, between two given profiles, the best alternatives of all individuals are identical, then the chosen alternatives share the same number of votes. Clearly, ETCD also has this property. Thus, ETCD has a weak relationship with anonymity, neutrality, and tops-only. Despite the relationship of the four conditions, they are logically independent under $(m, n)$ such that anonymity and neutrality are incompatible. We show the independence in Appendix A.1.

\section{Tie-breaking plurality rule}

We introduce a class of social choice functions that satisfy ETCD: the class of tiebreaking plurality (TBP) rules. A TBP rule is a social choice function derived from the plurality rule in a natural way. That is, it always selects an alternative from the set of alternatives having the maximal votes by breaking a tie in some manner if there is more than one alternative in that set. Namely, it is a tie-breaking procedure of the plurality rule. Formally, a TBP rule is represented as a selection of the plurality rule, as defined below.

A tie-breaking plurality rule $f_{p}$ is a selection of $F_{p}$, i.e., for each $P \in \mathscr{P}^{N}$,

$$
f_{p}(P) \in F_{p}(P) .
$$

There are many ways to break a tie. The following examples ${ }^{12}$ are parts of the TBP rules.

Example 1 (Pazner and Wesley 1978) Let $X=\left\{x_{1}, \ldots, x_{m}\right\}$. A TBP rule $f_{1}$ is defined by for each $P \in \mathscr{P}^{N}$,

$$
f_{1}(P)=x_{k^{*}}, \text { where } k^{*}=\min _{k}\left\{k: x_{k} \in F_{p}(P)\right\} .
$$

$\overline{12 f_{8} \text { and } f_{9} \text { in Examples } 8 \text { and } 9}$ of Appendix A.1, respectively, are also examples of TBP rules. 
$f_{1}$ chooses an alternative having the smallest index number among alternatives having the maximal votes. That is, the smaller the index number, the higher its priority. $f_{1}$ satisfies anonymity and tops-only, but violates neutrality.

Example 2 A TBP rule $f_{2}$ is defined by for each $P=\left(P_{i}\right)_{i \in N} \in \mathscr{P}^{N}$,

$$
f_{2}(P)=t\left(P_{j^{*}}\right), \text { where } j^{*}=\min _{j}\left\{j: j \in \bigcup_{x \in F_{p}(P)} N(x, P)\right\} .
$$

$f_{2}$ chooses an alternative that is most preferred for an individual having the smallest index number among individuals whose top-ranked alternatives have maximal votes. $f_{2}$ satisfies neutrality and tops-only, but violates anonymity.

Example 3 Let $(m, n)$ with $m \geq 3$ be such that every prime factor of $n$ exceeds $m .^{13}$ A TBP rule $f_{3}$ in this example is a simple application of the elimination method of the Coombs social choice function. Given $P \in \mathscr{P}^{N}$ and a nonempty $Y \subseteq X$, let $L(P, Y) \subseteq Y$ be the set of alternatives that are last-ranked over $Y$ by the largest number of individuals under $P$. It is known that the assumption of $(m, n)$ implies that $L(P, Y) \subsetneq Y$ whenever $|Y| \geq 2 .{ }^{14}$ Thus, a sequence $\left\{B_{t}\right\}$ defined by

$$
B_{0}=F_{p}(P), B_{1}=B_{0} \backslash L\left(P, B_{0}\right), B_{2}=B_{1} \backslash L\left(P, B_{1}\right), \ldots, B_{t}=B_{t-1} \backslash L\left(P, B_{t-1}\right), \ldots
$$

satisfies $\left|B_{T}\right|=1$ for some $T$. Let $f_{3}(P)=B_{T} \cdot f_{3}(P)$ is the result of extraction from $F_{p}(P)$ by the elimination method of the Coombs social choice function. ${ }^{15} f_{3}$ satisfies anonymity and neutrality, but violates tops-only.

It is obvious that all TBP rules satisfy ETCD, monotonicity, and, hence, weak monotonicity. On the other hand, some TBP rules satisfy some of the three conditions (anonymity, neutrality, or tops-only), whereas others violate some of them. Generally, as shown in the following proposition, even if $(m, n)$ satisfies the condition of Fact 2 (i.e., even if anonymity and neutrality are compatible on $(m, n)$ ), it is impossible for any TBP rule on $(m, n)$ to satisfy all of the three conditions.

Proposition 4 Assume that $(m, n)$ with $m \geq 3$ is such that anonymity and neutrality are compatible. Then, there is no tie-breaking plurality rule that satisfies anonymity, neutrality, and tops-only.

The proof is in Appendix B.4. This proposition indicates that for any $(m, n)$ such that anonymity and neutrality are compatible, even with TBP rules, it is impossible to satisfy a more demanding property than anonymity and neutrality. Not

\footnotetext{
13 Recall that this condition implies that anonymity and neutrality are compatible (see footnote 11).

14 For the details, see Moulin (1980) and (1983).

15 In the definition of $f_{3}$, if for all $P \in \mathscr{P}^{N}, F_{p}(P)$ is replaced with $X$, then $f_{3}$ coincides with the Coombs social choice function.
} 
surprisingly, this impossibility is only a trivial issue under the incompatibility problem of anonymity and neutrality, and is not a critical issue for TBP rules. TBP rules have the great advantage that they can be employed in any situation of $(m, n)$ while satisfying ETCD, which plays the role of equal treatment conditions both for individuals and for alternatives. As the main results of the paper, it is shown that the class of TBP rules is the unique class of social choice functions that satisfy ETCD and monotonicity conditions. We provide the characterizations in the next section. TBP rules are also justified from different viewpoints in Sect. 6.

\section{Characterization}

The class of TBP rules is characterized by ETCD and monotonicity conditions. The following result is our first characterization.

Theorem 1 A social choice function $f$ satisfies equal treatment of congruent distributions and monotonicity if and only if it is one of the tie-breaking plurality rules.

Corollary 1 Assume that $m=2$ and $n$ is odd. Then, a social choice function $f$ satisfies anonymity, neutrality, and weak monotonicity if and only if it is one of the tiebreaking plurality rules. ${ }^{16}$

The independence of axioms is given in Appendix A.2. The proof of Theorem 1 is in Appendix C.1. Corollary 1 follows from Proposition 1 and the fact that monotonicity coincides with weak monotonicity whenever $m=2$. As another corollary, even under $(m, n)$ such that anonymity and neutrality are compatible, it is shown that no social choice function completely inherits the properties of the plurality rule.

Corollary 2 Assume that $(m, n)$ with $m \geq 3$ is such that anonymity and neutrality are compatible. Then, there is no social choice function that satisfies anonymity, neutrality, tops-only, and monotonicity.

Proof of Corollary 2: Suppose that $f$ satisfies anonymity, neutrality, tops-only, and monotonicity. By Proposition 2, $f$ also satisfies ETCD. Thus, by Theorem $1, f$ is a TBP rule that satisfies anonymity, neutrality, and tops-only. However, this is a contradiction to Proposition 4.

Although our characterization in Theorem 1 is valid for all $n \geq 3$, monotonicity can be replaced with weak monotonicity in large finite societies. The following results are our second characterizations by ETCD and weak monotonicity.

Theorem 2 The following holds:

\footnotetext{
${ }^{16}$ In this case, each TBP rule is identical to majority voting (with no tie). This result is a variation of May (1952).
} 
[1] Assume that $m \leq 4$. Then, a social choice function $f$ satisfies equal treatment of congruent distributions and weak monotonicity if and only if it is one of the tiebreaking plurality rules.

[2] Assume that $m \geq 5$. Then, there exists $\bar{n}>m$ such that if $n \geq \bar{n}$, then a social choice function $f$ satisfies equal treatment of congruent distributions and weak monotonicity if and only if it is one of the tie-breaking plurality rules.

The proof of Theorem 2 is given in Appendix C.2. The statement [1] states that if $m \leq 4$, then for all $n$, the class of TBP rules is characterized by ETCD and weak monotonicity. On the other hand, the statement [2] states that even if $m \geq 5$, for all sufficiently large $n$, the similar characterization holds.

Remark 1 Note that the assumption of $(m, n)$ in [1] (together with $n \geq 3$ ) is a special case of $(m, n)$ with $m \leq n+1$. [1] indicates that for all $(m, n)$ with $m \leq \min \{4, n+1\}$, the characterization by ETCD and weak monotonicity is valid. However, it remains unclear whether the class of TBP rules is characterized by ETCD and weak monotonicity for all $(m, n)$ with $5 \leq m \leq n+1$. As a side note, more generally, it is shown, in Lemma 2 in Appendix C.2, that under $(m, n)$ with $m \leq n+2$, if a social choice function satisfies ETCD and weak monotonicity, then the chosen alternative at a given profile belongs to the set of top-ranked alternatives, which is a necessary condition to be a TBP rule. It may be possible to characterize the class of TBP rules by ETCD and weak monotonicity for all $(m, n)$ with $m \leq n+2$. We leave it as an open question. On the other hand, for some $(m, n)$ with $m>n+2$, there exists a social choice function that satisfies ETCD and weak monotonicity, but it is different from any TBP rule, as shown in the below example.

Example 4 Suppose either that $n$ is odd and $m \geq n+4$ or that $n$ is even and $m \geq n+3$. Let $X=\left\{x_{1}, \ldots, x_{m}\right\}$. A social choice function $f_{4}$ is defined by for each $P=\left(P_{i}\right)_{i \in N} \in \mathscr{P}^{N}$,

$$
f_{4}(P)=\left\{\begin{array}{l}
F_{p}(P), \text { if }\left|F_{p}(P)\right|=1, \\
x_{k^{*}}, \text { where } k^{*}=\min _{k}\left\{k: x_{k} \in F_{p}(P)\right\}, \text { if }\left|F_{p}(P)\right| \geq 2 \text { and }\left|X_{0}(P)\right| \leq n, \text { and } \\
x_{k^{*}}, \text { where } k^{*}=\min _{k}\left\{k: x_{k} \in X_{0}(P) \backslash\left\{t_{2}\left(P_{1}\right), \ldots, t_{2}\left(P_{n}\right)\right\}\right\}, \text { otherwise. }
\end{array}\right.
$$

Note that the third case of $f_{4}$ is possible. For example, if $n$ is odd and equal to $2 k+1$ for some integer $k \geq 1$, then let $P=\left(P_{i}\right)_{i \in N} \in \mathscr{P}^{N}$ be such that for all $j \in\{1, \ldots, k\}, t\left(P_{j}\right)=x_{1}$, for all $j \in\{k+1, \ldots, 2 k\}, t\left(P_{j}\right)=x_{2}$, and $t\left(P_{2 k+1}\right)=x_{3}$. Note $^{17}$ then that $\left|F_{p}(P)\right|=2$ or 3 and $\left|X_{0}(P)\right|=m-3>n$. The last inequality follows from the assumption of $m \geq n+4$. Furthermore, $\left|X_{0}(P)\right|>n$ implies that $\left|X_{0}(P) \backslash\left\{t_{2}\left(P_{1}\right), \ldots, t_{2}\left(P_{n}\right)\right\}\right|=\left|X_{0}(P)\right|-\left|\left\{t_{2}\left(P_{1}\right), \ldots, t_{2}\left(P_{n}\right)\right\}\right| \geq\left|X_{0}(P)\right|-n>0$. Thus, $X_{0}(P) \backslash\left\{t_{2}\left(P_{1}\right), \ldots, t_{2}\left(P_{n}\right)\right\}$ is nonempty, and so $f_{4}(P)$ is well-defined. By $f_{4}(P) \notin F_{p}(P), f_{4}$ is different from any TBP rule. It is similar for the case that $n$ is even and $m \geq n+3$. Then, it is checked that $f_{4}$ satisfies ETCD and weak

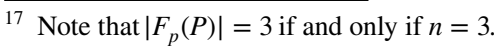


monotonicity. ETCD follows from the facts that for all $P, P^{\prime} \in \mathscr{P}^{N}$ having CD, $\left|F_{p}(P)\right|=1$ if and only if $\left|F_{p}\left(P^{\prime}\right)\right|=1,\left[\left|F_{p}(P)\right| \geq 2\right.$ and $\left.\left|X_{0}(P)\right| \leq n\right]$ if and only if $\left[\left|F_{p}\left(P^{\prime}\right)\right| \geq 2\right.$ and $\left.\left|X_{0}\left(P^{\prime}\right)\right| \leq n\right]$, and $\left[\left|F_{p}(P)\right| \geq 2\right.$ and $\left.\left|X_{0}(P)\right|>n\right]$ if and only if $\left[\left|F_{p}\left(P^{\prime}\right)\right| \geq 2\right.$ and $\left.\left|X_{0}\left(P^{\prime}\right)\right|>n\right]$. For weak monotonicity, given $P=\left(P_{i}\right)_{i \in N} \in \mathscr{P}^{N}$ and $j \in N$, let $t_{2}\left(P_{j}\right)=f_{4}(P)$. By the definition of $f_{4}$, we have either (i) $\left|F_{p}(P)\right|=1$ or (ii) $\left[\left|F_{p}(P)\right| \geq 2\right.$ and $\left.\left|X_{0}(P)\right| \leq n\right]$. Let $P^{\prime}=\left(\tilde{P}\left(P_{j} ; f_{4}(P)\right), P_{-j}\right)$. Both of (i) and (ii) imply that $F_{p}\left(P^{\prime}\right)=\left\{f_{4}(P)\right\}$, and so $f_{4}\left(P^{\prime}\right)=F_{p}\left(P^{\prime}\right)=f_{4}(P)$ for both (i) and (ii).

\section{Concluding remarks}

We have introduced a condition called ETCD. ETCD requires some invariance between two profiles having CD. ETCD is a conditional generalization of anonymity and neutrality on the situations in which those conditions are compatible. Furthermore, since ETCD implies ANV and NNV, which are weak equal treatment conditions for individuals and for alternatives, respectively, a social choice function satisfying ETCD treats individuals equally to some extent and alternatives equally to some extent even on the situations in which anonymity and neutrality are incompatible. Then, we provided two characterizations of the class of TBP rules by ETCD and two mild positive responsiveness conditions. These results indicate that TBP rules are supported from two viewpoints of equal treatment and positive responsiveness criteria. Furthermore, it is obvious that any TBP rule satisfies efficiency. That is, the chosen alternative at a given profile is not Pareto-dominated. Thus, TBP rules are also supported from the viewpoint of efficiency criteria.

Furthermore, TBP rules are partially supported from the viewpoint of incentive compatibility criteria by the following two aspects: First, by the result of Pazner and Wesley (1978), TBP rules ${ }^{18}$ are approximately strategy-proof ${ }^{19}$ in situations where the number of individuals is sufficiently large. Second, Campbell and Kelly (2016) characterized the (single-valued) plurality rule by strategy-proofness, nondictatorship, and citizen sovereignty ${ }^{20}$ on the resolute domain on which the set of alternatives with the maximal votes is always a singleton. That is, the plurality rule is necessarily single-valued on the resolute domain. Our domain includes the resolute domain, and TBP rules coincide with the plurality rule on the resolute domain. Thus, TBP rules are immune to strategic behavior on the resolute domain. These two arguments partially support TBP rules from the viewpoint of incentive compatibility criteria.

We leave some open questions for future research. First, as mentioned in the previous section, it remains unsolved whether the class of TBP rules is characterized by

\footnotetext{
18 They employed a TBP rule $f_{1}$ in Example 1. However, the similar proof can be applied to all TBP rules.

19 Strategy-proofness, which is an incentive compatibility condition, requires that truth-telling is a weak dominant strategy for any individual.

${ }^{20}$ Citizen sovereignty, which is a weaker efficiency condition, requires that every alternative should be chosen under some profiles (i.e., a social choice function is surjective).
} 
ETCD and weak monotonicity for all $(m, n)$ with $m \leq n+2$. Second, in our setting, the numbers of alternatives and individuals are fixed. On the other hand, some studies $^{21}$ employ the settings in which the numbers of alternatives and/or individuals vary. ETCD can be redefined in such settings in a suitable way. It may be possible to characterize TBP rules by ETCD and some consistency axioms in those settings. Third, our results are characterizations of the class of TBP rules, not a characterization of a specific TBP rule. Thus, our research is a starting point of analyzing specific tie-breaking rules in the class of TBP rules. It is future research to characterize some reasonable TBP rules as a further step. Finally, although ETCD was defined as a condition on social choice functions, it can be easily extended to social choice correspondences, as follows:

ETCD (for social choice correspondences): For each $P \in \mathscr{P}^{N}$ and each $P^{\prime} \in \mathscr{P}^{N}$, if $P$ and $P^{\prime}$ have congruent distributions, then for all $\ell \in\{0,1, \ldots, n\}$, $\left|F(P) \cap X_{\ell}(P)\right|=\left|F\left(P^{\prime}\right) \cap X_{\ell}\left(P^{\prime}\right)\right|$.

Note that it coincides with ETCD used in the previous sections whenever $F$ is always single-valued. Clearly, the plurality rule satisfies this condition. A characterization of the plurality rule by this condition is also an open question.

\section{Appendix A: Independence of axioms}

\section{Appendix A.1 Independence of the four conditions under the incompatibility of anonymity and neutrality}

We show the independence of anonymity, neutrality, tops-only, and ETCD under $(m, n)$ such that anonymity and neutrality are incompatible. To do so, it suffices to check the following six cases, owing to the incompatibility of anonymity and neutrality:

- ETCD and Tops-only $\nRightarrow$ Anonymity (see Example 5)

- ETCD and Tops-only $\nRightarrow$ Neutrality (see Example 5)

- Anonymity and Tops-only $\nRightarrow$ ETCD (see Example 6)

- Neutrality and Tops-only $\nRightarrow$ ETCD (see Example 7)

- For $m \geq 3,{ }^{22}$ ETCD and Anonymity $\nRightarrow$ Tops-only (see Example 8)

- For $m \geq 3$, ETCD and Neutrality $\nRightarrow$ Tops-only (see Example 9)

\footnotetext{
${ }^{21}$ Examples of the setting with variable alternatives and variable population include Fishburn (1977), Richelson (1978), Ching (1996), Baharad and Nitzan (2005), Yeh (2006), and Öztürk (2020). Examples of the setting with variable alternatives include Tideman (1987), Zavist and Tideman (1989), Laffond et al. (1996), Laslier (1996) and (2000), and Schulze (2011). Examples of the setting with variable population include Young (1974) and (1975), Moulin (1988), Yeh (2008), and Sekiguchi (2012). All literature except for Schulze (2011) listed here is the one concerned with social choice correspondences or functions. Schulze's (2011) procedure is somewhat special and outputs both a single alternative and a strict partial order on the set of alternatives.

22 Recall that any social choice function satisfies tops-only whenever $m=2$.
} 
Although the purpose of the remainder of this appendix is to provide examples of social choice functions for verifying the above cases under the incompatibility of anonymity and neutrality, most arguments in the following examples hold, regardless of whether or not $(m, n)$ satisfies the condition of Fact 2.

Example 5 (ETCD and Tops-only $\nRightarrow$ anonymity or neutrality) Assume $(m, n)$ with the property either that $m \geq 3$ or that $m=2$ and $n$ is even (hence $n \geq 4$ ). ${ }^{23}$ Let $X=\left\{x_{1}, x_{2}, \ldots, x_{m}\right\}$. A social choice function $f_{5}$ is defined by for each $P=\left(P_{i}\right)_{i \in N} \in \mathscr{P}^{N}$,

$$
f_{5}(P)=\left\{\begin{array}{l}
x_{k^{*}}, \text { where } k^{*}=\min _{k}\left\{k: x_{k} \in M(P)\right\}, \text { if } t\left(P_{1}\right)=x_{1} \text { or } t\left(P_{2}\right)=x_{1} \text { and } \\
x_{k^{*}}, \text { where } k^{*}=\max _{k}\left\{k: x_{k} \in M(P)\right\}, \text { otherwise, }
\end{array}\right.
$$

where $\quad M(P)=\left\{x_{k} \in X \backslash X_{0}(P):\left|N\left(x_{k}, P\right)\right| \leq\left|N\left(x_{k^{\prime}}, P\right)\right|\right.$ for all $\left.x_{k^{\prime}} \in X \backslash X_{0}(P)\right\}$. That is, if $x_{1}$ is most preferred for at least one of individuals 1 and 2 , then $f_{5}$ selects an alternative having the smallest index number among alternatives with the minimal votes in the set of top-ranked alternatives. If not, then $f_{5}$ selects an alternative that has the largest index number among the similar alternatives. It is easily checked that $f_{5}$ violates both anonymity and neutrality since it depends both on the names of the specific individuals and the names of alternatives. However, $f_{5}$ satisfies ETCD, which follows from the fact that for all $P, P^{\prime} \in \mathscr{P}^{N}$ having $\mathrm{CD}$, there exists $\ell^{*} \geq 1$ such that $X_{\ell^{*}}(P)=M(P)$ and $X_{\ell^{*}}\left(P^{\prime}\right)=M\left(P^{\prime}\right)$. It is clear that $f_{5}$ satisfies tops-only.

Example 6 (anonymity and tops-only $\nRightarrow$ ETCD) Given $x \in X$, a social choice function $f_{6}$ is defined by for each $P \in \mathscr{P}^{N}$,

$$
f_{6}(P)=x .
$$

That is, $f_{6}$ is constant. It is clear that $f_{6}$ satisfies anonymity and tops-only. To show that $f_{6}$ violates ETCD, let $P \in \mathscr{P}^{N}$ and $P^{\prime} \in \mathscr{P}^{N}$ be such that $X_{n}(P)=\{x\}$ and $X_{n}\left(P^{\prime}\right)=\{y\}$, where $y \neq x$. Then $P$ and $P^{\prime}$ have CD. Since $f(P)=x \in X_{n}(P)$ and $f\left(P^{\prime}\right)=x \in X_{0}\left(P^{\prime}\right), f_{6}$ violates ETCD.

Example 7 (neutrality and tops-only $\nRightarrow$ ETCD) Let a social choice function $f_{7}$ be dictatorial. Then $f_{7}$ satisfies neutrality and tops-only. By Fact 1, it violates ETCD.

Example 8 (ETCD and anonymity $\nRightarrow$ tops-only) Suppose that $m \geq 3$. Let $X=\left\{x_{1}, x_{2}, \ldots, x_{m}\right\}$. A social choice function $f_{8}$ is defined by for each $P=\left(P_{i}\right)_{i \in N} \in \mathscr{P}^{N}$

$$
\begin{aligned}
f_{8}(P)= & x_{k^{*}}, \text { where } k^{*}=\min _{k}\left\{k: x_{k} \in F_{p}(P)\right. \text { and } \\
& \left.\sum_{i \in N} b\left(x_{k}, P_{i}\right) \geq \sum_{i \in N} b\left(x_{k^{\prime}}, P_{i}\right) \text { for all } x_{k^{\prime}} \in F_{p}(P)\right\},
\end{aligned}
$$

\footnotetext{
${ }^{23}$ Note that $(2, n)$ does not satisfy the condition of Fact 2 if and only if $n$ is even.
} 
where $b\left(x_{k}, P_{i}\right)=m-t$ if $x_{k}$ is $t$ 'th ranked-alternative with respect to $P_{i}$, i.e., it is the Borda score of $x_{k}$ according to $P_{i} . f_{8}$ selects an alternative having the smallest index number among alternatives maximizing the sum of the Borda scores in the set of alternatives having the maximal votes. It is one of the TBP rules, thus it satisfies ETCD. It is easily checked that $f_{8}$ satisfies anonymity, but it violates tops-only.

Example 9 (ETCD and neutrality $\nRightarrow$ tops-only) Suppose that $m \geq 3$. A social choice function $f_{9}$ is defined by for each $P=\left(P_{i}\right)_{i \in N} \in \mathscr{P}^{N}$,

$$
f_{9}(P)=\left\{x \in F_{p}(P): y P_{1} x \text { for all } y \in F_{p}(P) \backslash\{x\}\right\} .
$$

$f_{9}$ selects an alternative that is worst for individual 1 among alternatives having the maximal votes. It is also one of the TBP rules, and it satisfies ETCD. It satisfies neutrality, but violates tops-only.

\section{Appendix A.2 Independence of axioms in Theorem 1}

We check the independence of axioms in Theorem 1. Note that $f_{5}$ in Example 5 is well-defined for all $(m, n)$ (even if $m=2$ and $n$ is odd). Then, it satisfies ETCD, but it violates monotonicity. On the other hand, $f_{6}$ and $f_{7}$ in Examples 6 and 7, respectively, satisfy monotonicity, but they violate ETCD.

\section{Appendix B Proofs of Propositions}

\section{Appendix B.1 Proof of Proposition 1}

("If" part) Suppose that $f$ satisfies ETCD. Let $P \in \mathscr{P}^{N}$ and $P^{\prime} \in \mathscr{P}^{N}$ satisfy the assumption of anonymity. It is obvious that $P$ and $P^{\prime}$ have CD. Since $m=2$ and $n$ is odd, there is no tie with respect to the number of votes. That is, there are $\ell, \ell^{\prime} \in\{0,1, \ldots, n\}$ with $\ell \neq \ell^{\prime}$ such that $\ell+\ell^{\prime}=n, X_{\ell}(P)=X_{\ell}\left(P^{\prime}\right)$ with cardinality one, and $X_{\ell^{\prime}}(P)=X_{\ell^{\prime}}\left(P^{\prime}\right)$ with cardinality one. Let $X_{\ell}(P) \equiv\{x\}$ and let $X_{\ell^{\prime}}(P) \equiv\{y\}$. By ETCD, it holds that $f(P)=x$ if and only if $f\left(P^{\prime}\right)=x$ and that $f(P)=y$ if and only if $f\left(P^{\prime}\right)=y$. Thus, we have $f(P)=f\left(P^{\prime}\right)$.

Next, let $P=\left(P_{i}\right)_{i \in N} \in \mathscr{P}^{N}$ and $P^{\prime}=\left(P_{i}^{\prime}\right)_{i \in N} \in \mathscr{P}^{N}$ satisfy the assumption of neutrality, i.e., there exists a bijection $\sigma: X \rightarrow X$ such that for all $i \in N$ and for all $a, b \in X, a P_{i} b$ if and only if $\sigma(a) P_{i}^{\prime} \sigma(b)$. Note that $P$ and $P^{\prime}$ have CD. Since $m=2$ and $n$ is odd, there are $\ell, \ell^{\prime} \in\{0,1, \ldots, n\}$ with $\ell \neq \ell^{\prime}$ such that $\ell+\ell^{\prime}=n$, $\left|X_{\ell}(P)\right|=\left|X_{\ell}\left(P^{\prime}\right)\right|=1$, and $\left|X_{\ell^{\prime}}(P)\right|=\left|X_{\ell^{\prime}}\left(P^{\prime}\right)\right|=1$. Let $X_{\ell}(P) \equiv\{x\}$ and let $X_{\ell^{\prime}}(P) \equiv\{y\}$. Then, by the definition of $\sigma$, it must be the case that $\{\sigma(x)\}=X_{\ell}\left(P^{\prime}\right)$ and $\{\sigma(y)\}=X_{\ell^{\prime}}\left(P^{\prime}\right)$. By ETCD, it holds that $f(P)=x$ if and only if $f\left(P^{\prime}\right)=\sigma(x)$ and that $f(P)=y$ if and only if $f\left(P^{\prime}\right)=\sigma(y)$. Thus, we have $\sigma(f(P))=f\left(P^{\prime}\right)$.

("Only if" part) Suppose that $f$ satisfies anonymity and neutrality and that $P=\left(P_{i}\right)_{i \in N} \in \mathscr{P}^{N}$ and $P^{\prime}=\left(P_{i}^{\prime}\right)_{i \in N} \in \mathscr{P}^{N}$ have CD. Since $m=2$ and $n$ is odd, there 
are $\ell, \ell^{\prime} \in\{0,1, \ldots, n\}$ with $\ell \neq \ell^{\prime}$ such that $\ell+\ell^{\prime}=n,\left|X_{\ell}(P)\right|=\left|X_{\ell}\left(P^{\prime}\right)\right|=1$, and $\left|X_{\ell^{\prime}}(P)\right|=\left|X_{\ell^{\prime}}\left(P^{\prime}\right)\right|=1$. Let $X_{\ell}(P) \equiv\{x\}$ and let $X_{\ell^{\prime}}(P) \equiv\{y\}$. There are two cases.

Case $1\left[X_{\ell}\left(P^{\prime}\right)=\{x\}\right]$ : Let a bijection $\pi: N \rightarrow N$ be such that for all $j \in N(x, P)$, $\pi(j) \in N\left(x, P^{\prime}\right)$ (hence, for all $j \in N(y, P), \pi(j) \in N\left(y, P^{\prime}\right)$ ). Let $P^{\prime \prime}=\left(P_{i}^{\prime \prime}\right)_{i \in N} \in \mathscr{P}^{N}$ be defined by for all $j \in N, P_{\pi(j)}^{\prime \prime}=P_{j}$. By anonymity, we have $f(P)=f\left(P^{\prime \prime}\right)$. On the other hand, by $m=2$, it must be the case of $P^{\prime \prime}=P^{\prime}$, which implies that $f\left(P^{\prime \prime}\right)=f\left(P^{\prime}\right)$. Therefore, we have $f(P)=f\left(P^{\prime \prime}\right)=f\left(P^{\prime}\right)$, meaning that $f(P)=x$ if and only if $f\left(P^{\prime}\right)=x$ and that $f(P)=y$ if and only if $f\left(P^{\prime}\right)=y$.

Case $2\left[X_{\ell}\left(P^{\prime}\right)=\{y\}\right]$ : Let a bijection $\pi^{\prime}: N \rightarrow N$ be such that for all $j \in N(x, P)$, $\pi^{\prime}(j) \in N\left(y, P^{\prime}\right)$ (and so, for all $j \in N(y, P), \pi^{\prime}(j) \in N\left(x, P^{\prime}\right)$ ). Then, we define $P^{\prime \prime \prime}=\left(P_{i}^{\prime \prime \prime}\right)_{i \in N} \in \mathscr{P}^{N}$ by for all $j \in N, P_{\pi^{\prime}(j)}^{\prime \prime \prime}=P_{j}$. Anonymity implies that $f(P)=f\left(P^{\prime \prime \prime}\right)$. On the other hand, $P^{\prime \prime \prime}$ and $P^{\prime}$ satisfies the assumption of neutrality by a bijection $\sigma: X \rightarrow X$ such that $\sigma(x)=y$ and $\sigma(y)=x$. Neutrality then implies that $\sigma\left(f\left(P^{\prime \prime \prime}\right)\right)=f\left(P^{\prime}\right)$. Thus, we have $f(P) \neq \sigma(f(P))=\sigma\left(f\left(P^{\prime \prime \prime}\right)\right)=f\left(P^{\prime}\right)$, meaning that $f(P)=x$ if and only if $f\left(P^{\prime}\right)=y$ and that $f(P)=y$ if and only if $f\left(P^{\prime}\right)=x$.

\section{Appendix B.2 Proof of Proposition 2}

Suppose that $f$ satisfies anonymity, neutrality and tops-only. Let $P=\left(P_{i}\right)_{i \in N} \in \mathscr{P}^{N}$ and $P^{\prime}=\left(P_{i}^{\prime}\right)_{i \in N} \in \mathscr{P}^{N}$ have CD. Let a bijection $\sigma: X \rightarrow X$ be such that

for all $\ell \in\{0,1, \ldots, n\}$ with $X_{\ell}(P) \neq \emptyset$ and all $x \in X_{\ell}(P), \sigma(x) \in X_{\ell}\left(P^{\prime}\right)$.

Since $P$ and $P^{\prime}$ have CD, such $\sigma$ exists. Let a bijection $\pi: N \rightarrow N$ be such that

for all $x \in X$ with $N(x, P) \neq \emptyset$ and all $j \in N(x, P), \pi(j) \in N\left(\sigma(x), P^{\prime}\right)$.

By the definition of $\sigma$, such $\pi$ exists. Let $P^{\prime \prime}=\left(P_{i}^{\prime \prime}\right)_{i \in N} \in \mathscr{P}^{N}$ be defined by for all $j \in N, \quad P_{\pi(j)}^{\prime \prime}=P_{j}$. By anonymity, we have $f(P)=f\left(P^{\prime \prime}\right)$. Next, let $P^{\prime \prime \prime}=\left(P_{i}^{\prime \prime \prime}\right)_{i \in N} \in \mathscr{P}^{N}$ be defined by for all $j \in N$ and all $x, y \in X, \sigma(x) P_{j}^{\prime \prime \prime} \sigma(y)$ if and only if $x P_{j}^{\prime \prime} y$. Then, neutrality implies that $\sigma\left(f\left(P^{\prime \prime}\right)\right)=f\left(P^{\prime \prime \prime}\right)$. On the other hand, note that for all $j \in N, t\left(P_{j}^{\prime}\right)=t\left(P_{j}^{\prime \prime \prime}\right)$. Thus, tops-only implies that $f\left(P^{\prime}\right)=f\left(P^{\prime \prime \prime}\right)$. The relations derived the above imply that $\sigma(f(P))=\sigma\left(f\left(P^{\prime \prime}\right)\right)=f\left(P^{\prime \prime \prime}\right)=f\left(P^{\prime}\right)$. Given $\ell \in\{0,1, \ldots, n\}$ with $X_{\ell}(P) \neq \emptyset$, recall that for all $x \in X_{\ell}(P), \sigma(x) \in X_{\ell}\left(P^{\prime}\right)$. Therefore, $f(P) \in X_{\ell}(P)$ if and only if $f\left(P^{\prime}\right)=\sigma(f(P)) \in X_{\ell}\left(P^{\prime}\right)$.

\section{Appendix B.3 Proof of Proposition 3}

We show only the latter parts of [1]-[3]. Each proof is almost identical to the proof of Proposition 1.

[1] Suppose that $f$ satisfies $\mathrm{ANV}$ and that $P \in \mathscr{P}^{N}$ and $P^{\prime} \in \mathscr{P}^{N}$ satisfy the assumption of anonymity. $P$ and $P^{\prime}$ then satisfy the assumption of ANV as well. 
Since $m=2$ and $n$ is odd, there are $\ell, \ell^{\prime} \in\{0,1, \ldots, n\}$ with $\ell \neq \ell^{\prime}$ such that $\ell+\ell^{\prime}=n, X_{\ell}(P)=X_{\ell}\left(P^{\prime}\right)$ with cardinality one, and $X_{\ell^{\prime}}(P)=X_{\ell^{\prime}}\left(P^{\prime}\right)$ with cardinality one. Let $X_{\ell}(P) \equiv\{x\}$ and let $X_{\ell^{\prime}}(P) \equiv\{y\}$. By ANV, it holds that $f(P)=x$ if and only if $f\left(P^{\prime}\right)=x$ and that $f(P)=y$ if and only if $f\left(P^{\prime}\right)=y$. Thus, we have $f(P)=f\left(P^{\prime}\right)$.

[2] Suppose that $f$ satisfies NNV and that $P=\left(P_{i}\right)_{i \in N} \in \mathscr{P}^{N}$ and $P^{\prime}=\left(P_{i}^{\prime}\right)_{i \in N} \in \mathscr{P}^{N}$ satisfy the assumption of neutrality, i.e., there exists a bijection $\sigma: X \rightarrow X$ such that for all $i \in N$ and for all $a, b \in X, a P_{i} b$ if and only if $\sigma(a) P_{i}^{\prime} \sigma(b)$. $P$ and $P^{\prime}$ then satisfy the assumption of NNV as well. Since $m=2$ and $n$ is odd, there are $\ell, \ell^{\prime} \in\{0,1, \ldots, n\}$ with $\ell \neq \ell^{\prime}$ such that $\ell+\ell^{\prime}=n,\left|X_{\ell}(P)\right|=\left|X_{\ell}\left(P^{\prime}\right)\right|=1$, and $\left|X_{\ell^{\prime}}(P)\right|=\left|X_{\ell^{\prime}}\left(P^{\prime}\right)\right|=1$. Let $X_{\ell}(P) \equiv\{x\}$ and let $X_{\ell^{\prime}}(P) \equiv\{y\}$. Then, by the definition of $\sigma$, it must be the case that $\{\sigma(x)\}=X_{\ell}\left(P^{\prime}\right)$ and $\{\sigma(y)\}=X_{\ell^{\prime}}\left(P^{\prime}\right)$. By $\mathrm{NNV}$, it holds that $f(P)=x$ if and only if $f\left(P^{\prime}\right)=\sigma(x)$ and that $f(P)=y$ if and only if $f\left(P^{\prime}\right)=\sigma(y)$. Thus, we have $\sigma(f(P))=f\left(P^{\prime}\right)$.

[3] Suppose that $f$ satisfies ANV and NNV. If $n$ is odd, then the result follows from [1], [2], and Proposition 1. Next, let $n$ be even. Let $P=\left(P_{i}\right)_{i \in N} \in \mathscr{P}^{N}$ and $P^{\prime}=\left(P_{i}^{\prime}\right)_{i \in N} \in \mathscr{P}^{N}$ have $\mathrm{CD}$. Then, there exists $\ell \in\{1, \ldots, n\}$ such that $\left|X_{\ell}(P)\right|=\left|X_{\ell}\left(P^{\prime}\right)\right| \in\{1,2\}$. If $\left|X_{\ell}(P)\right|=\left|X_{\ell}\left(P^{\prime}\right)\right|=2$, meaning that $X_{\ell}(P)=X_{\ell}\left(P^{\prime}\right)=X$, then the result is obvious. If $\left|X_{\ell}(P)\right|=\left|X_{\ell}\left(P^{\prime}\right)\right|=1$, then for $\ell^{\prime} \equiv n-\ell \geq 0$ with $\ell^{\prime} \neq \ell$, we have $\left|X_{\ell^{\prime}}(P)\right|=\left|X_{\ell^{\prime}}\left(P^{\prime}\right)\right|=1$. Let $X_{\ell}(P) \equiv\{x\}$ and let $X_{\ell^{\prime}}(P) \equiv\{y\}$. There are two cases: the case of $X_{\ell}\left(P^{\prime}\right)=\{x\}$ and the case of $X_{\ell}\left(P^{\prime}\right)=\{y\}$.

Case $1\left[X_{\ell}\left(P^{\prime}\right)=\{x\}\right]$ : This case immediately implies that there is a bijection $\pi: N \rightarrow N$ such that for each $j \in N, P_{\pi(j)}^{\prime}=P_{j}$. Thus, by ANV, it holds that $f(P)=x$ if and only if $f\left(P^{\prime}\right)=x$ and that $f(P)=y$ if and only if $f\left(P^{\prime}\right)=y$.

Case $2\left[X_{\ell}\left(P^{\prime}\right)=\{y\}\right]$ : Let a bijection $\pi^{\prime}: N \rightarrow N$ be such that for all $j \in N(x, P)$, $\pi^{\prime}(j) \in N\left(y, P^{\prime}\right) \quad$ and so, for all $\left.j \in N(y, P), \quad \pi^{\prime}(j) \in N\left(x, P^{\prime}\right)\right)$. We define $P^{\prime \prime}=\left(P_{i}^{\prime \prime}\right)_{i \in N} \in \mathscr{P}^{N}$ by for all $j \in N, P_{\pi^{\prime}(j)}^{\prime \prime}=P_{j}$. Note that $X_{\ell}(P)=X_{\ell}\left(P^{\prime \prime}\right)=\{x\}$ (and so, $\left.X_{\ell^{\prime}}(P)=X_{\ell^{\prime}}\left(P^{\prime \prime}\right)=\{y\}\right)$. ANV then implies that $f(P)=x$ if and only if $f\left(P^{\prime \prime}\right)=x$ and that $f(P)=y$ if and only if $f\left(P^{\prime \prime}\right)=y$. Thus, we have $f(P)=f\left(P^{\prime \prime}\right)$. On the other hand, $P^{\prime \prime}$ and $P^{\prime}$ satisfies the assumption of NNV by a bijection $\sigma: X \rightarrow X$ such that $\sigma(x)=y$ and $\sigma(y)=x$. NNV then implies that $f\left(P^{\prime \prime}\right)=x$ if and only if $f\left(P^{\prime}\right)=y$ and that $f\left(P^{\prime \prime}\right)=y$ if and only if $f\left(P^{\prime}\right)=x$. Thus, we have $\sigma\left(f\left(P^{\prime \prime}\right)\right)=f\left(P^{\prime}\right)$. Hence, we have $f(P) \neq \sigma(f(P))=\sigma\left(f\left(P^{\prime \prime}\right)\right)=f\left(P^{\prime}\right)$, meaning that $f(P)=x$ if and only if $f\left(P^{\prime}\right)=y$ and that $f(P)=y$ if and only if $f\left(P^{\prime}\right)=x$.

\section{Appendix B.4 Proof of Proposition 4}

Let $f_{p}$ be a TBP rule. Suppose the contrary that $f_{p}$ satisfies anonymity, neutrality, and tops-only simultaneously. By the condition of Fact 2, $m \geq 3$ implies that $n \geq 4$. We show only the case that $n$ is odd since the similar argument applies in the case that $n$ is even. Let an integer $k \geq 2$ satisfy $n=2 k+1$. Given $a, b, c \in X$ that are different from each other, let $P=\left(P_{i}\right)_{i \in N} \in \mathscr{P}^{N}$ be such that

for all $j \in\{1, \ldots, k\}, t\left(P_{j}\right)=a$, for all $j \in\{k+1, \ldots, 2 k\}, t\left(P_{j}\right)=b$, and $t\left(P_{n}\right)=c$. 
Since $f_{p}$ is a TBP rule, we have $f_{p}(P) \in\{a, b\}$. Let a bijection $\sigma: X \rightarrow X$ be defined by $\sigma(a)=b, \sigma(b)=a$, and for all $x \neq a, b, \sigma(x)=x$. Let $P^{\prime}=\left(P_{i}^{\prime}\right)_{i \in N} \in \mathscr{P}^{N}$ be such that for all $j \in N$ and all $x, y \in X, \sigma(x) P_{j}^{\prime} \sigma(y)$ if and only if $x P_{j} y$. Neutrality then implies that $f_{p}\left(P^{\prime}\right)=\sigma\left(f_{p}(P)\right) \neq f_{p}(P)$. Next, let a bijection $\pi: N \rightarrow N$ be defined by

for all $j \in\{1, \ldots, k\}, \pi(j)=k+j$, for all $j \in\{k+1, \ldots, 2 k\}, \pi(j)=j-k$, and $\pi(n)=n$.

Let $P^{\prime \prime}=\left(P_{i}^{\prime \prime}\right)_{i \in N} \in \mathscr{P}^{N}$ be such that for all $j \in N, P_{\pi(j)}^{\prime \prime}=P_{j}^{\prime}$. Anonymity then implies that $f_{p}\left(P^{\prime}\right)=f_{p}\left(P^{\prime \prime}\right)$. On the other hand, tops-only implies that $f_{p}(P)=f_{p}\left(P^{\prime \prime}\right)$. Thus, $f_{p}(P)=f_{p}\left(P^{\prime \prime}\right)=f_{p}\left(P^{\prime}\right)=\sigma\left(f_{p}(P)\right) \neq f_{p}(P)$, a contradiction.

\section{Appendix C Proofs of Theorems}

In some of the following proofs, we implicitly use the following obvious fact about the plurality rule: For each $P \in \mathscr{P}^{N}$ and each $\bar{\ell} \in\{1, \ldots, n\}$ with $X_{\bar{\ell}}(P) \neq \emptyset$,

$$
F_{p}(P)=X_{\bar{\ell}}(P) \text { if and only if for all } \ell \in\{1, \ldots, n\} \text { with } X_{\ell}(P) \neq \emptyset, \bar{\ell} \geq \ell .
$$

\section{Appendix C. 1 Proof of Theorem 1}

Before the proof of Theorem 1, we define a notation. Given $P=\left(P_{i}\right)_{i \in N} \in \mathscr{P}^{N}$, $j, j^{\prime} \in N$, and $P_{j}^{\prime}, P_{j^{\prime}}^{\prime} \in \mathscr{P}$, we write a profile $\left(P_{j}^{\prime}, P_{j^{\prime}}^{\prime},\left(P_{i}\right)_{i \neq j, j^{\prime}}\right) \in \mathscr{P}^{N}$ as $\left(P_{j}^{\prime}, P_{j^{\prime}}^{\prime}, P_{-\left\{j j^{\prime}\right\}}\right)$. For the proof of Theorem 1, it suffices to show the "only if" part since it is self-evident that a TBP rule satisfies ETCD and monotonicity. Suppose that $f$ satisfies ETCD and monotonicity. Let $P=\left(P_{i}\right)_{i \in N} \in \mathscr{P}^{N}$ be given. We show that $f(P) \in F_{p}(P)$. The proof is divided into two steps.

Step 1. It holds that $f(P) \in X \backslash X_{0}(P){ }^{24}$

Since it is obvious for the case of $X_{0}(P)=\emptyset$, let $X_{0}(P) \neq \emptyset$. Suppose the contrary that $f(P) \in X_{0}(P)$. Let $x \in X \backslash X_{0}(P)$ be given. Then, $x \in X_{\ell^{\prime}}(P)$ for some $\ell^{\prime} \geq 1$, i.e., $|N(x, P)|=\ell^{\prime}$. Let $P^{\prime}=\left(P_{i}^{\prime}\right)_{i \in N} \in \mathscr{P}^{N}$ be defined by

for all $j \in N(x, P), P_{j}^{\prime}=\tilde{P}\left(P_{j} ; f(P)\right)$ and for all $j \in N \backslash N(x, P), P_{j}^{\prime}=P_{j}$.

Given $h \in N(x, P)$, monotonicity implies that $f\left(P_{h}^{\prime}, P_{-h}\right)=f(P)$. Furthermore, given $h^{\prime} \in N(x, P) \backslash\{h\}$ (if such exists), monotonicity also implies that $f\left(P_{h}^{\prime}, P_{h^{\prime}}^{\prime}, P_{-\left\{h, h^{\prime}\right\}}\right)=f\left(P_{h}^{\prime}, P_{-h}\right)=f(P)$. By repeating this argument, we have $f\left(P^{\prime}\right)=f(P) \in X_{\ell^{\prime}}\left(P^{\prime}\right)$. Note that $X_{\ell^{\prime}}\left(P^{\prime}\right)=\{f(P)\} \cup X_{\ell^{\prime}}(P) \backslash\{x\}$ and that for all $\ell \neq \ell^{\prime}, X_{\ell}\left(P^{\prime}\right)=X_{\ell}(P)$. These relations imply that for all $\ell \geq 0,\left|X_{\ell}\left(P^{\prime}\right)\right|=\left|X_{\ell}(P)\right|$. Thus, $P^{\prime}$ and $P$ have CD. However, we have $f\left(P^{\prime}\right) \in X_{\ell^{\prime}}\left(P^{\prime}\right)$ and $f(P) \in X_{0}(P)$, a contradiction to ETCD. This completes the proof of Step 1.

${ }^{24}$ This statement means that ETCD and monotonicity imply efficiency. 
Step 2. It holds that $f(P) \in F_{p}(P)$.

Let $\ell^{*}$ be such that $F_{p}(P)=X_{\ell^{*}}(P)$. By Step 1, it is obvious for the case that $X_{\ell^{*}}(P)=X \backslash X_{0}(P)$. Next, consider the remaining case that $X_{\ell^{*}}(P) \subsetneq X \backslash X_{0}(P)$. Suppose the contrary that $f(P) \equiv x \notin X_{\ell^{*}}(P)$. By Step 1, it holds that $x \in X_{\bar{\ell}}(P)$ for some $\bar{\ell} \geq 1$. Note that $\ell^{*}>\bar{\ell}$. Let $y \in X_{\ell^{*}}(P)$ be given and let $N^{\prime} \subset N(y, P)$ with $\left|N^{\prime}\right|=\ell^{*}-\bar{\ell}$ be given. Let $P^{\prime}=\left(P_{i}^{\prime}\right)_{i \in N} \in \mathscr{P}^{N}$ be defined by

$$
\text { for all } j \in N^{\prime}, P_{j}^{\prime}=\tilde{P}\left(P_{j} ; x\right) \text { and for all } j \notin N^{\prime}, P_{j}^{\prime}=P_{j} \text {. }
$$

Given $h \in N^{\prime}$, monotonicity implies that $f\left(P_{h}^{\prime}, P_{-h}\right)=x$. Furthermore, given $h^{\prime} \in N^{\prime} \backslash\{h\}$ (if such exists), monotonicity also implies that $f\left(P_{h}^{\prime}, P_{h^{\prime}}^{\prime}, P_{-\left\{h, h^{\prime}\right\}}\right)=f\left(P_{h}^{\prime}, P_{-h}\right)=x$. By repeating this argument, we have $f\left(P^{\prime}\right)=x \in X_{\ell^{*}}\left(P^{\prime}\right)$ and $y \in X_{\bar{\ell}}\left(P^{\prime}\right)$. It then holds the following relations: (i) $X_{\ell^{*}}\left(P^{\prime}\right)=\{x\} \cup X_{\ell^{*}}(P) \backslash\{y\}$, (ii) $X_{\bar{\ell}}\left(P^{\prime}\right)=\{y\} \cup X_{\bar{\ell}}(P) \backslash\{x\}$, and (iii) for all $\ell \neq \bar{\ell}, \ell^{*}, X_{\ell}\left(P^{\prime}\right)=X_{\ell}(P)$. (i)-(iii) imply that for all $\ell \geq 0,\left|X_{\ell}\left(P^{\prime}\right)\right|=\left|X_{\ell}(P)\right|$. Thus, $P^{\prime}$ and $P$ have CD. However, we have $f(P) \in X_{\bar{\ell}}(P)$ and $f\left(P^{\prime}\right) \in X_{\ell^{*}}\left(P^{\prime}\right)$, a contradiction to ETCD.

\section{Appendix C.2 Proof of Theorem 2}

In this appendix, we prove the "only if" parts of [1] and [2] of Theorem 2 with a series of lemmas. The case of $m=2$ in [1] follows from Theorem 1 with the fact that weak monotonicity is equivalent to monotonicity whenever $m=2$. Thus, the remaining case in [1] is $3 \leq m \leq 4$. Additionally, as a matter of fact, the proof of [2] can be also applied to not only the case of $m \geq 5$, but also to that of $3 \leq m \leq 4$. $^{25}$ Thus, we consider the case of $m \geq 3$ in the following. Throughout this appendix, we fix ( $m, n)$ with $m \geq 3$ (and, of course, $n \geq 3$ ) and assume that a social choice function $f$ satisfies ETCD and weak monotonicity.

We frequently use the following notation. For given $i \in N, P_{i} \in \mathscr{P}$, and $x \in X$, let $\tilde{P}_{2}\left(P_{i} ; x\right) \in \mathscr{P}$ be defined by $t_{2}\left(\tilde{P}_{2}\left(P_{i} ; x\right)\right)=x$ and for each $y, z \in X \backslash\{x\}, y \tilde{P}_{2}\left(P_{i} ; x\right) z$ if and only if $y P_{i} z$. That is, $\tilde{P}_{2}\left(P_{i} ; x\right)$ is a transformation of $P_{i}$ obtained by moving $x$ to the second rank and by preserving the relative rank of alternatives other than $x$.

Lemma 1 For each $P \in \mathscr{P}^{N}$ and each $\ell \geq 1$, if $f(P) \in X_{\ell}(P)$ and $f(P) \notin F_{p}(P)$, then $\left|X_{\ell}(P)\right| \geq 2$.

Proof of Lemma 1: Suppose the contrary that there exists $P=\left(P_{i}\right)_{i \in N} \in \mathscr{P}^{N}$ and $\bar{\ell} \geq 1$ such that $f(P) \equiv x \in X_{\bar{\ell}}(P), x \notin F_{p}(P)$, and $\left|X_{\bar{\ell}}(P)\right|=1$, i.e., $X_{\bar{\ell}}(P)=\{x\}$. By $x \notin F_{p}(P)$, there is $y \in X \backslash X_{0}(P)$ such that $|N(y, P)| \equiv \ell^{\prime}>\bar{\ell}$. Let $N^{\prime} \subset N(y, P)$ with $\left|N^{\prime}\right|=\ell^{\prime}-\bar{\ell}$ be given. Let $P^{\prime}=\left(P_{i}^{\prime}\right)_{i \in N} \in \mathscr{P}^{N}$ be defined by

$$
\text { for all } j \in N^{\prime}, P_{j}^{\prime}=\tilde{P}_{2}\left(P_{j} ; x\right) \text { and for all } j \in N \backslash N^{\prime}, P_{j}^{\prime}=P_{j} \text {. }
$$

\footnotetext{
${ }^{25}$ Due to the statement [1], it is not essential to mention the case of $3 \leq m \leq 4$ in the statement [2]. Thus, its mention has been omitted in [2].
} 
Note that for all $j \in N, t\left(P_{j}\right)=t\left(P_{j}^{\prime}\right)$, which implies that $X_{\bar{\ell}}\left(P^{\prime}\right)=\{x\}$ and that $P$ and $P^{\prime}$ have CD. Thus, by ETCD, it holds that $f\left(P^{\prime}\right) \in X_{\bar{\ell}}\left(P^{\prime}\right)$, i.e., $f\left(P^{\prime}\right)=x$. Next, let $P^{\prime \prime}=\left(P_{i}^{\prime \prime}\right)_{i \in N} \in \mathscr{P}^{N}$ be defined by

$$
\text { for all } j \in N^{\prime}, P_{j}^{\prime \prime}=\tilde{P}\left(P_{j}^{\prime} ; x\right) \text { and for all } j \in N \backslash N^{\prime}, P_{j}^{\prime \prime}=P_{j}^{\prime} \text {. }
$$

By weak monotonicity, given $h \in N^{\prime}$, we have $f\left(P_{h}^{\prime \prime}, P_{-h}^{\prime}\right)=x$. Furthermore, given $h^{\prime} \in N^{\prime} \backslash\{h\} \quad$ (if such exists), weak monotonicity also implies that $f\left(P_{h}^{\prime \prime}, P_{h^{\prime}}^{\prime \prime}, P_{-\left\{h, h^{\prime}\right\}}^{\prime}\right)=f\left(P_{h}^{\prime \prime}, P_{-h}^{\prime}\right)=x$. By repeating this argument, we have $f\left(P^{\prime \prime}\right)=x \in X_{\ell^{\prime}}\left(P^{\prime \prime}\right)$. Then, it can be checked that $P^{\prime \prime}$ and $P$ have $\mathrm{CD}$. At first, by the definition of $P^{\prime \prime}$, we have $X \backslash X_{0}\left(P^{\prime \prime}\right)=X \backslash X_{0}(P)$, which means that $X_{0}\left(P^{\prime \prime}\right)=X_{0}(P)$. Furthermore, by the definition of $P^{\prime \prime}$, it holds that (i) $\left|N\left(x, P^{\prime \prime}\right)\right|=|N(y, P)|$, (ii) $\left|N\left(y, P^{\prime \prime}\right)\right|=|N(x, P)|$, and (iii) for all $z \neq x, y$ with $z \notin X_{0}\left(P^{\prime \prime}\right)\left(=X_{0}(P)\right)$, $N\left(z, P^{\prime \prime}\right)=N(z, P)$. (i)-(iii) imply that for all $\ell \geq 0,\left|X_{\ell}\left(P^{\prime \prime}\right)\right|=\left|X_{\ell}(P)\right|$. Thus, $P^{\prime \prime}$ and $P$ have CD. However, we have $f(P) \in X_{\bar{\ell}}(P)$ and $f\left(P^{\prime \prime}\right) \in X_{\ell^{\prime}}\left(P^{\prime \prime}\right)$, a contradiction.

Lemma 2 If $m \leq n+2$, then for each $P \in \mathscr{P}^{N}, f(P) \in X \backslash X_{0}(P)$.

Proof of Lemma 2: Suppose that $m \leq n+2$. The proof consists of two steps: A and B. Step A is further divided into several steps.

Step A. For all $P \in \mathscr{P}^{N}$ with $\left|X \backslash X_{0}(P)\right| \geq 2, f(P) \in X \backslash X_{0}(P)$.

Suppose the contrary that $f\left(P^{0}\right) \in X_{0}\left(P^{0}\right)$ for some $P^{0} \in \mathscr{P}^{N}$ with $\left|X \backslash X_{0}\left(P^{0}\right)\right| \equiv k \geq 2$. Then, $1 \leq\left|X_{0}\left(P^{0}\right)\right|=m-k \leq m-2 \leq n$. The last inequality follows from $m \leq n+2$. Let $N^{0} \equiv\{1,2, \ldots, m-k\} \subseteq N$. Let a bijection $g: N^{0} \rightarrow X_{0}(P)$ be given. Let $P^{0 *}=\left(P_{i}^{0 *}\right)_{i \in N} \in \mathscr{P}^{N}$ be defined by

$$
\text { for all } j \in N^{0}, P_{j}^{0}=\tilde{P}_{2}\left(P_{j}^{0} ; g(j)\right) \text { and for all } j \notin N^{0}, P_{j}^{0 *}=P_{j}^{0} \text {. }
$$

Then since for all $j \in N, t\left(P_{j}^{0}\right)=t\left(P_{j}^{0 *}\right), P^{0}$ and $P^{0 *}$ have CD. Thus, by ETCD, we have $f\left(P^{0 *}\right) \in X_{0}\left(P^{0 *}\right)=X_{0}\left(P^{0}\right)$. By the definition of $P^{0 *}$, there is $i^{0 *} \in N^{0}$ such that $t_{2}\left(P_{i^{0 *}}^{0 *}\right)=g\left(i^{0 *}\right)=f\left(P^{0 *}\right)$. Let $t\left(P_{i^{0 *}}^{0 *}\right) \equiv \hat{x}$ and let $F_{p}\left(P^{0 *}\right) \equiv X_{\bar{\ell}}\left(P^{0 *}\right)$. In the following, despite $\hat{x} \in X \backslash X_{0}\left(P^{0 *}\right)$, it is shown that for all $\ell \in\{1, \ldots, \bar{\ell}\}, \hat{x} \notin X_{\ell}\left(P^{0 *}\right)$. If this fact is shown, then a contradiction is derived and the proof of Step A is complete. We prove this fact by induction on $\ell$.

Step A-1. It holds that $\hat{x} \notin X_{1}\left(P^{0 *}\right)$.

Let $P^{1} \equiv\left(\tilde{P}\left(P_{i 0 *}^{0 *} f\left(P^{0 *}\right)\right), P_{-i^{0 *}}^{0 *}\right) \in \mathscr{P}^{N}$. Weak monotonicity implies that $f\left(P^{1}\right)=f\left(P^{0 *}\right)$. Note that $f\left(P^{1}\right) \in X_{1}\left(P^{1}\right)$. Now, for the proof of $\hat{x} \notin X_{1}\left(P^{0 *}\right)$, suppose the contrary that $\hat{x} \in X_{1}\left(P^{0 *}\right)$. It is then shown that $P^{1}$ and $P^{0 *}$ have $\mathrm{CD}$. At first, it holds by the definitions of $P^{1}$ and $P^{0 *}$ that $X_{1}\left(P^{1}\right)=\left\{f\left(P^{1}\right)\right\} \cup X_{1}\left(P^{0 *}\right) \backslash\{\hat{x}\}$, which implies that $\left|X_{1}\left(P^{1}\right)\right|=\left|X_{1}\left(P^{0 *}\right)\right|$. Furthermore, it holds that for all $\ell^{\prime} \geq 2$, $X_{\ell^{\prime}}\left(P^{1}\right)=X_{\ell^{\prime}}\left(P^{0 *}\right),{ }^{26}$ thus $\left|X_{\ell^{\prime}}\left(P^{1}\right)\right|=\left|X_{\ell^{\prime}}\left(P^{0 *}\right)\right|$. These relations must imply

${ }^{26}$ These sets may be empty. 
$\left|X_{0}\left(P^{1}\right)\right|=\left|X_{0}\left(P^{0 *}\right)\right|$. Thus, $P^{1}$ and $P^{0 *}$ have CD. However, we have $f\left(P^{1}\right) \in X_{1}\left(P^{1}\right)$ and $f\left(P^{0 *}\right) \in X_{0}\left(P^{0 *}\right)$, a contradiction to ETCD.

Step A-2. Given $\ell \in\{1, \ldots, \bar{\ell}-1\},{ }^{27}$ suppose that for all $\ell^{\prime} \in\{1, \ldots, \ell\}$, $\hat{x} \notin X_{\ell^{\prime}}\left(P^{0 *}\right)$. Then, $\hat{x} \notin X_{\ell+1}\left(P^{0 *}\right)$.

Given $\ell \in\{1, \ldots, \bar{\ell}-1\}$. The assumption of the induction implies that $\hat{x} \in \bigcup_{\ell^{\prime \prime} \geq \ell+1} X_{\ell^{\prime \prime}}\left(P^{0 *}\right)$. We divide this step into two steps.

Step A-2-1. Let $P^{1}$ be the profile defined in Step $A-1$. Then, for every $\ell^{\prime} \in\{1, \ldots, \ell\}$, there exist profiles $P^{2}, \ldots, P^{\ell^{\prime}+1} \in \mathscr{P}^{N}$ such that, for all $\ell^{\prime \prime} \in\left\{1, \ldots, \ell^{\prime}\right\}$,

(1) $\left\{f\left(P^{\ell^{\prime \prime}+1}\right)\right\}=X_{\ell^{\prime \prime}+1}\left(P^{\ell^{\prime \prime}+1}\right) \cap X_{\ell^{\prime \prime}}\left(P^{\ell^{\prime \prime}}\right)$,

(2) for some $x^{\ell^{\prime \prime}} \in X_{\ell^{\prime \prime}}\left(P^{\ell^{\prime \prime}}\right) \backslash\left\{f\left(P^{\ell^{\prime \prime}+1}\right)\right\},\left\{x^{\ell^{\prime \prime}}\right\}=X_{\ell^{\prime \prime}-1}\left(P^{\ell^{\prime \prime}+1}\right) \cap X_{\ell^{\prime \prime}}\left(P^{\ell^{\prime \prime}}\right)$, and

(3) for all $x \in X \backslash\left\{f\left(P^{\ell^{\prime \prime}+1}\right), x^{\ell^{\prime \prime}}\right\}, N\left(x, P^{\ell^{\prime \prime}+1}\right)=N\left(x, P^{\ell^{\prime \prime}}\right)$.

By induction on $\ell^{\prime}$. At first, let $\ell^{\prime}=1$. We show that there exists $P^{2} \in \mathscr{P}^{N}$ such that (1) $\left\{f\left(P^{2}\right)\right\}=X_{2}\left(P^{2}\right) \cap X_{1}\left(P^{1}\right)$, (2) for some $x^{1} \in X_{1}\left(P^{1}\right) \backslash\left\{f\left(P^{2}\right)\right\}$, $\left\{x^{1}\right\}=X_{0}\left(P^{2}\right) \cap X_{1}\left(P^{1}\right)$, and (3) for all $x \in X \backslash\left\{f\left(P^{2}\right), x^{1}\right\}, N\left(x, P^{2}\right)=N\left(x, P^{1}\right)$. Note that $\left|N\left(\hat{x}, P^{1}\right)\right|=\left|N\left(\hat{x}, P^{0 *}\right)\right|-1 \geq \ell \geq 1$. The first equality follows from the definition of $P^{1}$. The second-to-last inequality follows from $\hat{x} \in \bigcup_{\ell^{\prime \prime} \geq \ell+1} X_{\ell^{\prime \prime}}\left(P^{0 *}\right)$. We then show that $\left|X_{1}\left(P^{1}\right)\right| \geq 2$. Recall that $f\left(P^{1}\right) \in X_{1}\left(P^{1}\right)$ and that $f\left(P^{1}\right) \neq \hat{x}$. Thus, if $\left|N\left(\hat{x}, P^{1}\right)\right|=1$ (i.e., $\left.\hat{x} \in X_{1}\left(P^{1}\right)\right)$, then we have $\left|X_{1}\left(P^{1}\right)\right| \geq 2$. On the other hand, if $\left|N\left(\hat{x}, P^{1}\right)\right|>1$ (i.e., $\bigcup_{\ell^{\prime \prime} \geq 2} X_{\ell^{\prime \prime}}\left(P^{1}\right) \neq \emptyset$ ), then we have $f\left(P^{1}\right) \notin F_{p}\left(P^{1}\right)$. Lemma 1 with $f\left(P^{1}\right) \in X_{1}\left(P^{1}\right)$ implies that $\left|X_{1}\left(P^{1}\right)\right| \geq 2$.

Now, let $N^{1} \equiv\left\{j \in N: t\left(P_{j}^{1}\right)=x\right.$ for some $\left.x \in X_{1}\left(P^{1}\right)\right\}$. Note that $\left|N^{1}\right|=\left|X_{1}\left(P^{1}\right)\right| \geq 2$. Thus, there exists a bijection $g_{1}: N^{1} \rightarrow X_{1}\left(P^{1}\right)$ such that for all $j \in N^{1}, g_{1}(j) \neq t\left(P_{j}^{1}\right)$. Let $P^{1 *}=\left(P_{i}^{1 *}\right)_{i \in N} \in \mathscr{P}^{N}$ be defined by

$$
\text { for all } j \in N^{1}, P_{j}^{1 *}=\tilde{P}_{2}\left(P_{j}^{1} ; g_{1}(j)\right) \text { and for all } j \notin N^{1}, P_{j}^{1 *}=P_{j}^{1} .
$$

Since for all $j \in N, t\left(P_{j}^{1}\right)=t\left(P_{j}^{1 *}\right), P^{1}$ and $P^{1 *}$ have CD. Thus, by ETCD with $f\left(P^{1}\right) \in X_{1}\left(P^{1}\right)$, we have $f\left(P^{1 *}\right) \in X_{1}\left(P^{1 *}\right)$. Since $X_{1}\left(P^{1 *}\right)=X_{1}\left(P^{1}\right)$, there is $i^{1 *} \in N^{1}$ such that $t_{2}\left(P^{1 *}\right)=g_{1}\left(i^{1 *}\right)=f\left(P^{1 *}\right)$.

Then, let $P^{2} \equiv\left(\tilde{P}\left(P_{i^{1 *}}^{i^{1 *}} f\left(P^{1 *}\right)\right), P_{-i^{1 *}}^{1 *}\right) \in \mathscr{P}^{N}$. Weak monotonicity implies that $f\left(P^{2}\right)=f\left(P^{1 *}\right)$. Note that $f\left(P^{2}\right) \in X_{2}^{-1 *}\left(P^{2}\right)$. It is then checked that $P^{2}$ satisfies (1)(3). At first, (1) is obvious. For (2), let $x^{1} \equiv t\left(P_{i^{1 *}}^{1 *}\right)$. Then, we have $x^{1} \neq f\left(P^{2}\right)$ and $\left\{x^{1}\right\}=X_{0}\left(P^{2}\right) \cap X_{1}\left(P^{1}\right)$. (3) is obvious. This completes the proof of the first part of the induction of Step A-2-1.

Next, we show the second part of the induction of Step A-2-1. Given $\ell^{\prime} \in\{1, \ldots, \ell-1\}$, suppose that there exist $P^{2}, \ldots, P^{\ell^{\prime}+1} \in \mathscr{P}^{N}$ such that for all $\ell^{\prime \prime} \in\left\{1, \ldots, \ell^{\prime}\right\}$,

${ }^{27}$ In this step, it is implicitly assumed that $\bar{\ell} \geq 2$. The proof of the case of $\bar{\ell}=1$ is complete by Step A-1. 
(1) $\left\{f\left(P^{\ell^{\prime \prime}+1}\right)\right\}=X_{\ell^{\prime \prime}+1}\left(P^{\ell^{\prime \prime}+1}\right) \cap X_{\ell \prime \prime}\left(P^{\ell^{\prime \prime}}\right)$,

(2) for some $x^{\ell^{\prime \prime}} \in X_{\ell^{\prime \prime}}\left(P^{\ell^{\prime \prime}}\right) \backslash\left\{f\left(P^{\ell^{\prime \prime}+1}\right)\right\},\left\{x^{\ell^{\prime \prime}}\right\}=X_{\ell^{\prime \prime}-1}\left(P^{\ell^{\prime \prime}+1}\right) \cap X_{\ell^{\prime \prime}}\left(P^{\ell^{\prime \prime}}\right)$, and

(3) for all $x \in X \backslash\left\{f\left(P^{\ell^{\prime \prime}+1}\right), x^{\ell^{\prime \prime}}\right\}, N\left(x, P^{\ell^{\prime \prime}+1}\right)=N\left(x, P^{\ell^{\prime \prime}}\right)$.

To complete the proof of this part, it suffices to show that there exists $P^{\ell^{\prime}+2} \in \mathscr{P}^{N}$ such that

(1) $)^{\prime} \quad\left\{f\left(P^{\ell^{\prime}+2}\right)\right\}=X_{\ell^{\prime}+2}\left(P^{\ell^{\prime}+2}\right) \cap X_{\ell^{\prime}+1}\left(P^{\ell^{\prime}+1}\right)$,

(2) ${ }^{\prime}$ for some $x^{\ell^{\prime}+1} \in X_{\ell^{\prime}+1}\left(P^{\ell^{\prime}+1}\right) \backslash\left\{f\left(P^{\ell^{\prime}+2}\right)\right\},\left\{x^{\ell^{\prime}+1}\right\}=X_{\ell^{\prime}}\left(P^{\ell^{\prime}+2}\right) \cap X_{\ell^{\prime}+1}\left(P^{\ell^{\prime}+1}\right)$, and

(3) for all $x \in X \backslash\left\{f\left(P^{\ell^{\prime}+2}\right), x^{\ell^{\prime}+1}\right\}, N\left(x, P^{\ell^{\prime}+2}\right)=N\left(x, P^{\ell^{\prime}+1}\right)$.

First of all, we show that $\hat{x} \neq f\left(P^{\ell^{\prime}+1}\right), x^{\ell^{\prime}}$. By (1) and (2), it holds that $f\left(P^{2}\right) \in X_{2}$ $\left(P^{2}\right), \ldots, f\left(P^{\ell^{\prime}}\right) \in X_{\ell^{\prime}}\left(P^{\ell^{\prime}}\right)$ and that $x^{1} \in X_{0}\left(P^{2}\right), x^{2} \in X_{1}\left(P^{3}\right), \ldots, x^{\ell^{\prime}-1} \in X_{\ell^{\prime}-2}\left(P^{\ell^{\prime}}\right)$. Thus, by (3), for all $\tilde{\ell}>\ell^{\prime}, \quad X_{\tilde{\ell}}\left(P^{\ell^{\prime}}\right)=\cdots=X_{\tilde{\ell}}\left(P^{2}\right)=X_{\tilde{\ell}}\left(P^{1}\right)$. Note that $\hat{\ell} \equiv\left|N\left(\hat{x}, P^{1}\right)\right|=\left|N\left(\hat{x}, P^{0 *}\right)\right|-1 \geq \ell>\ell^{\prime}$. The second-to-last inequality follows from $\hat{x} \in \bigcup_{\ell^{\prime \prime} \geq \ell+1} X_{\ell^{\prime \prime}}\left(P^{0 *}\right)$, and the last inequality follows from $\ell^{\prime} \in\{1, \ldots, \ell-1\}$. Thus, $\hat{x} \in X_{\hat{\ell}}\left(P^{1}\right)=X_{\hat{\ell}}\left(P^{2}\right)=\cdots=X_{\hat{\ell}}\left(P^{\ell^{\prime}}\right)$. Now, for the proof of $\hat{x} \neq f\left(P^{\ell^{\prime}+1}\right), x^{\ell^{\prime}}$, note, by (1) and (2), that $f\left(P^{\ell^{\prime}+1}\right) \in X_{\ell^{\prime}}\left(P^{\ell^{\prime}}\right)$ and $x^{\ell^{\prime}} \in X_{\ell^{\prime}}\left(P^{\ell^{\prime}}\right)$. On the other hand, by $\hat{x} \in X_{\hat{\ell}}\left(P^{\ell^{\prime}}\right)$ with $\hat{\ell} \neq \ell^{\prime}$, we have $\hat{x} \neq f\left(P^{\ell^{\prime}+1}\right), x^{\ell^{\prime}}$.

Secondly, we show that $\left|X_{\ell^{\prime}+1}\left(P^{\ell^{\prime}+1}\right)\right| \geq 2$. Since $\hat{x} \neq f\left(P^{\ell^{\prime}+1}\right), x^{\ell^{\prime}}$, by (3) with the above arguments, we have $\left|N\left(\hat{x}, P^{\ell^{\prime}+1}\right)\right|=\left|N\left(\hat{x}, P^{\ell^{\prime}}\right)\right|=\hat{\ell} \geq \ell \geq \ell^{\prime}+1$. If $\left|N\left(\hat{x}, P^{\ell^{\prime}+1}\right)\right|=\ell^{\prime}+1$ (i.e., $\left.\hat{x} \in X_{\ell^{\prime}+1}\left(P^{\ell^{\prime}+1}\right)\right)$, then we have $\left|X_{\ell^{\prime}+1}\left(P^{\ell^{\prime}+1}\right)\right| \geq 2$ since $f\left(P^{\ell^{\prime}+1}\right) \in X_{\ell^{\prime}+1}\left(P^{\ell^{\prime}+1}\right)$ and since $f\left(P^{\ell^{\prime}+1}\right) \neq \hat{x}$. On the other hand, if $\left|N\left(\hat{x}, P^{\ell^{\prime}+1}\right)\right|>\ell^{\prime}+1 \quad$ (i.e., $\left.\quad \bigcup_{\ell^{\prime \prime} \geq \ell^{\prime}+2} X_{\ell^{\prime \prime}}\left(P^{\ell^{\prime}+1}\right) \neq \emptyset\right)$, then we have $f\left(P^{\ell^{\prime}+1}\right) \notin F_{p}\left(P^{\ell^{\prime}+1}\right)$. Lemma 1 then implies that $\left|X_{\ell^{\prime}+1}\left(P^{\ell^{\prime}+1}\right)\right| \geq 2$.

Now, let $N^{\ell^{\prime}+1} \subset N$ with $\left|N^{\ell^{\prime}+1}\right|=\left|X_{\ell^{\prime}+1}\left(P^{\ell^{\prime}+1}\right)\right|$ be such that for all $j \in N^{\ell^{\prime}+1}$, $t\left(P_{j}^{\ell^{\prime}+1}\right) \in X_{\ell^{\prime}+1}\left(P^{\ell^{\prime}+1}\right)$ and for all $j, j^{\prime} \in N^{\ell^{\prime}+1}$ with $j \neq j^{\prime}, t\left(P_{j}^{\ell^{\prime}+1}\right) \neq t\left(P_{j^{\prime}}^{\ell^{\prime}+1}\right) .{ }^{28}$ Let a bijection $g_{\ell^{\prime}+1}: N^{\ell^{\prime}+1} \rightarrow X_{\ell^{\prime}+1}\left(P^{\ell^{\prime}+1}\right)$ be such that for all $j \in N^{\ell^{\prime}+1}$, $g_{\ell^{\prime}+1}(j) \neq t\left(P_{j}^{\ell^{\prime}+1}\right)$. By $\quad\left|X_{\ell^{\prime}+1}\left(P^{\ell^{\prime}+1}\right)\right| \geq 2$, such $\quad g_{\ell^{\prime}+1}$ exists. Then, let $P^{\left(\ell^{\prime}+1\right) *}=\left(P_{i}^{\left(\ell^{\prime}+1\right) *}\right)_{i \in N} \in \mathscr{P}^{N}$ be defined by

for all $j \in N^{\ell^{\prime}+1}, P_{j}^{\left(\ell^{\prime}+1\right) *}=\tilde{P}_{2}\left(P_{j}^{\ell^{\prime}+1} ; g_{\ell^{\prime}+1}(j)\right)$ and for all $j \notin N^{\ell^{\prime}+1}, P_{j}^{\left(\ell^{\prime}+1\right) *}=P_{j}^{\ell^{\prime}+1}$.

Since for all $i \in N, t\left(P_{j}^{\ell^{\prime}+1}\right)=t\left(P_{j}^{\left(\ell^{\prime}+1\right) *}\right), P^{\ell^{\prime}+1}$ and $P^{\left(\ell^{\prime}+1\right) *}$ have CD. Thus, by ETCD with $f\left(P^{\ell^{\prime}+1}\right) \in X_{\ell^{\prime}+1}\left(P^{\ell^{\prime}+1}\right)$, we have $f\left(P^{\left(\ell^{\prime}+1\right) *}\right) \in X_{\ell^{\prime}+1}\left(P^{\left(\ell^{\prime}+1\right) *}\right)$. Since $X_{\ell^{\prime}+1}\left(P^{\left(\ell^{\prime}+1\right) *}\right)=X_{\ell^{\prime}+1}\left(P^{\ell^{\prime}+1}\right)$, there is $i^{\left(\ell^{\prime}+1\right) *} \in N^{\ell^{\prime}+1}$ such that $t_{2}\left(P_{i\left(\ell^{\prime}+1\right) *}^{\left(\ell^{\prime}+1\right) *}\right)=g_{\ell^{\prime}+1}\left(i^{\left(\ell^{\prime}+1\right) *}\right)=f\left(P^{\left(\ell^{\prime}+1\right) *}\right)$.

Then, let $P^{\ell^{\prime}+2} \equiv\left(\tilde{P}\left(P_{i^{\left(\ell^{\prime}+1\right) *}}^{\left(\ell^{\prime}+1\right) *} ; f\left(P^{\left(\ell^{\prime}+1\right) *}\right)\right), P_{-i^{\left(\ell^{\prime}+1\right) *}}^{\left(\ell^{\prime}+1\right) *}\right) \in \mathscr{P}^{N}$. Weak monotonicity implies that $f\left(P^{\ell^{\prime}+2}\right)=f\left(P^{\left(\ell^{\prime}+1\right) *}\right)$. Note that $f\left(P^{\ell^{\prime}+2}\right) \in X_{\ell^{\prime}+2}\left(P^{\ell^{\prime}+2}\right)$. It is then checked that $P^{\ell^{\prime}+2}$ satisfies the properties $(1)^{\prime}-(3)^{\prime}$. At first, $(1)^{\prime}$ is obvious. For $(2)^{\prime}$, let $\quad x^{\ell^{\prime}+1} \equiv t\left(P_{i\left(\ell^{\prime}+1\right) *}^{\left(\ell^{\prime}+1\right) *}\right.$. Then, we have $x^{\ell^{\prime}+1} \neq f\left(P^{\ell^{\prime}+2}\right) \quad$ and

\footnotetext{
${ }^{28} N^{\ell^{\prime}+1}$ has the same role as $N^{1}$ in the first part of the induction of Step A-2-1. Since there is a one-toone correspondence between an individual and the top-ranked alternative for the individual in $X_{1}\left(P^{1}\right)$, the definition of $N^{1}$ is simpler than the one of $N^{\ell^{\prime}+1}$.
} 
$\left\{x^{\ell^{\prime}+1}\right\}=X_{\ell^{\prime}}\left(P^{\ell^{\prime}+2}\right) \cap X_{\ell^{\prime}+1}\left(P^{\ell^{\prime}+1}\right)$. (3)' is obvious. This completes the proof of Step A-2-1.

Step A-2-2. It holds that $\hat{x} \notin X_{\ell+1}\left(P^{0 *}\right)$.

Let $P^{2}, \ldots, P^{\ell+1} \in \mathscr{P}^{N}$ satisfy (1)-(3) in Step A-2-1. It is then easily checked that the following relations hold: Given $\ell^{\prime} \in\{1, \ldots, \ell\}$,

$$
\begin{aligned}
X_{\ell^{\prime}+1}\left(P^{\ell^{\prime}+1}\right) & =\left\{f\left(P^{\ell^{\prime}+1}\right)\right\} \cup X_{\ell^{\prime}+1}\left(P^{\ell^{\prime}}\right), \\
X_{\ell^{\prime}}\left(P^{\ell^{\prime}+1}\right) & =X_{\ell^{\prime}}\left(P^{\ell^{\prime}}\right) \backslash\left\{f\left(P^{\ell^{\prime}+1}\right), x^{\ell^{\prime}}\right\}, \\
X_{\ell^{\prime}-1}\left(P^{\ell^{\prime}+1}\right) & =\left\{x^{\ell^{\prime}}\right\} \cup X_{\ell^{\prime}-1}\left(P^{\ell^{\prime}}\right), \text { and } \\
X_{\tilde{\ell}}\left(P^{\ell^{\prime}+1}\right) & =X_{\tilde{\ell}}\left(P^{\ell^{\prime}}\right) \text { for all } \tilde{\ell} \text { with } \tilde{\ell}>\ell^{\prime}+1 \text { or } \tilde{\ell}<\ell^{\prime}-1 .
\end{aligned}
$$

These relations are crucial for the proof of this step.

To show $\hat{x} \notin X_{\ell+1}\left(P^{0 *}\right)$, suppose the contrary that $\hat{x} \in X_{\ell+1}\left(P^{0 *}\right)$. It is then shown that $P^{\ell+1}$ and $P^{0 *}$ have CD. However, if this fact is shown, then a contradiction to ETCD is derived since $f\left(P^{\ell+1}\right) \in X_{\ell+1}\left(P^{\ell+1}\right)$ and $f\left(P^{0 *}\right) \in X_{0}\left(P^{0 *}\right)$. Thus, to complete the proof of Step A-2-2, we have only to show this fact. The proof of this fact is divided into two cases.

Case $1[\ell=1]$ : By the definition of $P^{1}$ and the above relations $(*)$ with $\hat{x} \in X_{2}\left(P^{0 *}\right)$, it holds the following relations:

$$
X_{2}\left(P^{1}\right)=X_{2}\left(P^{0 *}\right) \backslash\{\hat{x}\} \text { and } X_{2}\left(P^{2}\right)=\left\{f\left(P^{2}\right)\right\} \cup X_{2}\left(P^{1}\right) .
$$

These relations imply $\left|X_{2}\left(P^{2}\right)\right|=\left|X_{2}\left(P^{0 *}\right)\right|$. Furthermore, the following relations hold:

$$
X_{1}\left(P^{1}\right)=\left\{\hat{x}, f\left(P^{1}\right)\right\} \cup X_{1}\left(P^{0 *}\right) \text { and } X_{1}\left(P^{2}\right)=X_{1}\left(P^{1}\right) \backslash\left\{f\left(P^{2}\right), x^{1}\right\} .
$$

Thus, we have $\left|X_{1}\left(P^{2}\right)\right|=\left|X_{1}\left(P^{0 *}\right)\right|$. Finally, it holds that for all $\tilde{\ell} \geq 3$, $X_{\tilde{\ell}}\left(P^{2}\right)=X_{\tilde{\ell}}\left(P^{0 *}\right)$, thus $\left|X_{\tilde{\ell}}\left(P^{2}\right)\right|=\left|X_{\tilde{\ell}}\left(P^{0 *}\right)\right|$. The relations obtained in the above together imply $\left|X_{0}\left(P^{2}\right)\right|=\left|X_{0}\left(P^{0 *}\right)\right|$. Thus, $P^{2}$ and $P^{0 *}$ have CD.

Case $2[\ell \geq 2]$ : By the definition of $P^{1}$ and the above relations $(*)$ with $\hat{x} \in X_{\ell+1}\left(P^{0 *}\right)$, it holds the following relations:

$$
\begin{aligned}
X_{\ell+1}\left(P^{1}\right) & =X_{\ell+1}\left(P^{0 *}\right) \backslash\{\hat{x}\}, \\
X_{\ell+1}\left(P^{\ell}\right) & =\cdots=X_{\ell+1}\left(P^{1}\right), \text { and } \\
X_{\ell+1}\left(P^{\ell+1}\right) & =\left\{f\left(P^{\ell+1}\right)\right\} \cup X_{\ell+1}\left(P^{\ell}\right) .
\end{aligned}
$$

These relations imply that $\left|X_{\ell+1}\left(P^{\ell+1}\right)\right|=\left|X_{\ell+1}\left(P^{0 *}\right)\right|$. Next, the following relations hold: 


$$
\begin{aligned}
X_{\ell}\left(P^{1}\right) & =\{\hat{x}\} \cup X_{\ell}\left(P^{0 *}\right), \\
X_{\ell}\left(P^{\ell-1}\right) & =\cdots=X_{\ell}\left(P^{1}\right), \\
X_{\ell}\left(P^{\ell}\right) & =\left\{f\left(P^{\ell}\right)\right\} \cup X_{\ell}\left(P^{\ell-1}\right), \text { and } \\
X_{\ell}\left(P^{\ell+1}\right) & =X_{\ell}\left(P^{\ell}\right) \backslash\left\{f\left(P^{\ell+1}\right), x^{\ell}\right\} .
\end{aligned}
$$

These relations imply that $\left|X_{\ell}\left(P^{\ell+1}\right)\right|=\left|X_{\ell}\left(P^{0 *}\right)\right|$. Next, given $\ell^{\prime} \in\{1, \ldots, \ell-1\}$, the following relations ${ }^{29}$ hold:

$$
\begin{aligned}
X_{\ell^{\prime}}\left(P^{\ell^{\prime}-1}\right) & =\cdots=X_{\ell^{\prime}}\left(P^{0 *}\right), \\
X_{\ell^{\prime}}\left(P^{\ell^{\prime}}\right) & =\left\{f\left(P^{\ell^{\prime}}\right)\right\} \cup X_{\ell^{\prime}}\left(P^{\ell^{\prime}-1}\right), \\
X_{\ell^{\prime}}\left(P^{\ell^{\prime}+1}\right) & =X_{\ell^{\prime}}\left(P^{\ell^{\prime}}\right) \backslash\left\{f\left(P^{\ell^{\prime}+1}\right), x^{\ell^{\prime}}\right\}, \\
X_{\ell^{\prime}}\left(P^{\ell^{\prime}+2}\right) & =\left\{x^{\ell^{\prime}+1}\right\} \cup X_{\ell^{\prime}}\left(P^{\ell^{\prime}+1}\right), \text { and } \\
X_{\ell^{\prime}}\left(P^{\ell+1}\right) & =\cdots=X_{\ell^{\prime}}\left(P^{\ell^{\prime}+2}\right) .
\end{aligned}
$$

These relations imply that $\left|X_{\ell^{\prime}}\left(P^{\ell+1}\right)\right|=\left|X_{\ell^{\prime}}\left(P^{0 *}\right)\right|$. Finally, it holds that for all $\tilde{\ell} \geq \ell+2, X_{\tilde{\ell}}\left(P^{\ell+1}\right)=X_{\tilde{\ell}}\left(P^{0 *}\right)$, thus $\left|X_{\tilde{\ell}}\left(P^{\ell+1}\right)\right|=\left|X_{\tilde{\ell}}\left(P^{0 *}\right)\right|$. The relations obtained in the above together imply $\left|X_{0}\left(P^{\ell+1}\right)\right|=\left|X_{0}\left(P^{0 *}\right)\right|$. Thus, $P^{\ell+1}$ and $P^{0 *}$ have CD. This completes the proof of Step A-2-2, and hence, Step A.

Step B. For all $P \in \mathscr{P}^{N}$ with $\left|X \backslash X_{0}(P)\right|=1, f(P) \in X \backslash X_{0}(P)$.

Given $x, y \in X$ with $x \neq y$, let $\bar{P} \in \mathscr{P}^{N}$ be such that (i) $t\left(\bar{P}_{1}\right)=x$ and $t_{2}\left(\bar{P}_{1}\right)=y$, (ii) $t\left(\bar{P}_{2}\right)=y$ and $t_{2}\left(\bar{P}_{2}\right)=x$ and (iii) for all $j \neq 1,2, \bar{P}_{j}=\bar{P}_{2}$. By Step $\mathrm{A}$, $f(\bar{P}) \in\{x, y\}$. We show that $f(\bar{P})=y$. To show this, suppose that $f(\bar{P})=x$. Then, we let $\bar{P}^{\prime} \in \mathscr{P}^{N}$ be defined by $\bar{P}_{1}^{\prime}=\bar{P}_{1}$ and for all $j \neq 1, \bar{P}_{j}^{\prime}=\tilde{P}\left(\bar{P}_{j} ; x\right)$. It then holds by weak monotonicity that $f\left(\bar{P}_{2}^{\prime}, \bar{P}_{-2}\right)=x$. Furthermore, it implies that $f\left(\bar{P}_{2}^{\prime}, \bar{P}_{3}^{\prime}, \bar{P}_{-\{2,3\}}\right)=f\left(\bar{P}_{2}^{\prime}, \bar{P}_{-2}\right)=x$. By repeating this argument, we have $f\left(\bar{P}_{n}, \bar{P}_{-n}^{\prime}\right)=x$. Note that $\left(\bar{P}_{n}, \bar{P}_{-n}^{\prime}\right)$ and $\bar{P}$ have $\mathrm{CD}$. However, we have $f\left(\bar{P}_{n}, \bar{P}_{-n}^{\prime}\right) \in X_{n-1}\left(\bar{P}_{n}, \bar{P}_{-n}^{\prime}\right)$ and $f(\bar{P}) \in X_{1}(\bar{P})$. This is a contradiction to ETCD. Thus, $f(\bar{P})=y$. Then, by weak monotonicity, $f\left(\tilde{P}\left(\bar{P}_{1} ; y\right), \bar{P}_{-1}\right)=y$. Note that $\{y\}=X_{n}\left(\tilde{P}\left(\bar{P}_{1} ; y\right), \bar{P}_{-1}\right)$. Thus, for given $P \in \mathscr{P}^{N}$ with $\left|X \backslash X_{0}(P)\right|=1, P$ and $\left(\tilde{P}\left(\bar{P}_{1} ; y\right), \bar{P}_{-1}\right)$ have $\mathrm{CD}$, thus, by ETCD, we obtain $f(P) \in X_{n}(P)=X \backslash X_{0}(P)$.

Lemma 3 For each $P \in \mathscr{P}^{N}$ and each $\ell \geq 1$, if $f(P) \in X_{\ell}(P)$ and $f(P) \notin F_{p}(P)$, then $X_{\ell+1}(P) \neq \emptyset$.

Proof of Lemma 3: Let $P=\left(P_{i}\right)_{i \in N} \in \mathscr{P}^{N}$ and $\ell \geq 1$ be such that $f(P) \in X_{\ell}(P)$ and $f(P) \notin F_{p}(P)$. Suppose the contrary that $X_{\ell+1}(P)=\emptyset$. By Lemma $1,\left|X_{\ell}(P)\right| \geq 2$. Let $N^{\prime} \subset N$ with $\left|N^{\prime}\right|=\left|X_{\ell}(P)\right|$ be such that for all $j \in N^{\prime}, t\left(P_{j}\right) \in X_{\ell}(P)$ and that for all $h, h^{\prime} \in N^{\prime}, t\left(P_{h}\right) \neq t\left(P_{h^{\prime}}\right)$. Let a bijection $g: N^{\prime} \rightarrow X_{\ell}(P)$ be such that for all $j \in N^{\prime}, g(j) \neq t\left(P_{j}\right)$. Then let $P^{\prime}=\left(P^{\prime}\right)_{i \in N} \in \mathscr{P}^{N}$ be such that for each $j \in N^{\prime}$,

\footnotetext{
${ }^{29}$ Since $X_{1}\left(P^{0}\right)=X_{1}\left(P^{0 *}\right)$, the first relation is trivially true whenever $\ell^{\prime}=1$.
} 
$P_{j}^{\prime}=\tilde{P}_{2}\left(P_{j} ; g(j)\right)$ and that for each $j \notin N^{\prime}, P_{j}^{\prime}=P_{j}$. Then, since for all $j \in N$, $t\left(P_{j}\right)=t\left(P_{j}^{\prime}\right), P$ and $P^{\prime}$ have $\mathrm{CD}$. Thus, ETCD implies that $f\left(P^{\prime}\right) \equiv x \in X_{\ell}\left(P^{\prime}\right)$. Since $X_{\ell}\left(P^{\prime}\right)=X_{\ell}(P)$, there is $j^{\prime} \in N^{\prime} \quad$ such that $t_{2}\left(P_{j^{\prime}}^{\prime}\right)=g\left(j^{\prime}\right)=x$. Let $P^{\prime \prime} \equiv\left(\tilde{P}\left(P_{j^{\prime}}^{\prime} ; x\right), P_{-j^{\prime}}^{\prime}\right) \in \mathscr{P}^{N}$. By weak monotonicity, we have $f\left(P^{\prime \prime}\right)=x \in X_{\ell+1}\left(P^{\prime \prime}\right)$. Since $X_{\ell+1}\left(P^{\prime}\right)=X_{\ell+1}(P)=\emptyset$, it follows from the definition of $P^{\prime \prime}$ that $X_{\ell+1}\left(P^{\prime \prime}\right)=\{x\}$. Note that $f(P) \in X_{\ell}(P), f(P) \notin F_{p}(P)$, and $X_{\ell+1}(P)=\emptyset$ together imply that there exists $\ell^{\prime} \geq \ell+2$ such that $X_{\ell^{\prime}}(P) \neq \emptyset$, which in turn implies that $X_{\ell^{\prime}}\left(P^{\prime \prime}\right)=X_{\ell^{\prime}}\left(P^{\prime}\right) \neq \emptyset$. Thus, we have $x \notin F_{p}\left(P^{\prime \prime}\right)$, which, with $X_{\ell+1}\left(P^{\prime \prime}\right)=\{x\}$, derives a contradiction to Lemma 1.

Lemma 4 For each $P \in \mathscr{P}^{N}$ and each $\ell \geq 1$, if $f(P) \in X_{\ell}(P)$ and $f(P) \notin F_{p}(P)$, then $\left|X_{\ell}(P)\right|>\left|X_{\ell+1}(P)\right| \cdot(\ell+1)$.

Proof of Lemma 4: Let $P=\left(P_{i}\right)_{i \in N} \in \mathscr{P}^{N}$ and $\bar{\ell} \geq 1$ be such that $f(P) \in X_{\bar{\ell}}(P)$ and $f(P) \notin F_{p}(P)$. Suppose the contrary that $\left|X_{\bar{\ell}}(P)\right| \leq\left|X_{\bar{\ell}+1}(P)\right| \cdot(\bar{\ell}+1)$. Let $N^{\prime} \subset N$ with $\left|N^{\prime}\right|=\left|X_{\bar{\ell}}(P)\right|$ be such that for all $j \in N^{\prime}, \quad t\left(P_{j}\right) \in X_{\bar{\ell}+1}(P)$. By $\left|X_{\bar{\ell}}(P)\right| \leq\left|X_{\bar{\ell}+1}(P)\right| \cdot(\bar{\ell}+1)$, such $N^{\prime}$ exists. Let a bijection $g: N^{\prime} \rightarrow X_{\bar{\ell}}(P)$ be given. Let $P^{\prime}=\left(P_{i}^{\prime}\right)_{i \in N} \in \mathscr{P}^{N}$ be such that for all $j \in N^{\prime}, P_{j}^{\prime}=\tilde{P}_{2}\left(P_{j} ; g(j)\right)$ and that for all $j \notin N^{\prime}, P_{j}^{\prime}=P_{j}$. Then since for all $j \in N, t\left(P_{j}\right)=t\left(P_{j}^{\prime}\right), P$ and $P^{\prime}$ have $\mathrm{CD}$. Thus, we have $f\left(P^{\prime}\right) \equiv x \in X_{\bar{\ell}}\left(P^{\prime}\right)$. Since $X_{\bar{\ell}}\left(P^{\prime}\right)=X_{\bar{\ell}}(P)$, there is $j^{\prime} \in N^{\prime}$ such that $t_{2}\left(P_{j^{\prime}}^{\prime}\right)=g\left(j^{\prime}\right)=x$. Let $P^{\prime \prime} \equiv\left(\tilde{P}\left(P_{j^{\prime}}^{\prime} ; x\right), P_{-j^{\prime}}^{\prime}\right) \in \mathscr{P}^{N}$. Then weak monotonicity implies that $f\left(P^{\prime \prime}\right)=x \in X_{\bar{\ell}+1}\left(P^{\prime \prime}\right)$. Next, we show that $P^{\prime \prime}$ and $P^{\prime}$ have $\mathrm{CD}$. To do so, note that it holds the following relations: (i) $X_{\bar{\ell}+1}\left(P^{\prime \prime}\right)=\{x\} \cup X_{\bar{\ell}+1}\left(P^{\prime}\right) \backslash\left\{t\left(P_{j^{\prime}}^{\prime}\right)\right\}$, (ii) $X_{\bar{\ell}}\left(P^{\prime \prime}\right)=\left\{t\left(P_{j^{\prime}}^{\prime}\right)\right\} \cup X_{\bar{\ell}}\left(P^{\prime}\right) \backslash\{x\}$, and (iii) for all $\ell \notin\{\bar{\ell}, \bar{\ell}+1\}, X_{\ell}\left(P^{\prime \prime}\right)=X_{\ell}\left(P^{\prime}\right)$. (i)-(iii) imply that for all $\ell \geq 0,\left|X_{\ell}\left(P^{\prime \prime}\right)\right|=\left|X_{\ell}\left(P^{\prime}\right)\right|$. Thus, $P^{\prime \prime}$ and $P^{\prime}$ have $\mathrm{CD}$. However, we have $f\left(P^{\prime \prime}\right)=x \in X_{\ell+1}\left(P^{\prime \prime}\right)$ and $f\left(P^{\prime}\right)=x \in X_{\ell}\left(P^{\prime}\right)$, a contradiction.

We now turn to the proof of [1] of Theorem 2.

Proof of [1] of Theorem 2: Let $P \in \mathscr{P}^{N}$ be given. We show that $f(P) \in F_{p}(P)$. The assumption of $m \leq 4$ together with $n \geq 3$ implies that $(m, n)$ satisfies $m \leq n+2$. Thus, by Lemma 2, we have $f(P) \in X_{\ell}(P)$ for some $\ell \geq 1$. Suppose the contrary that $f(P) \notin F_{p}(P)$. By Lemmas 1 and 3 with $m \leq 4$, it holds that $\left|X_{\ell}(P)\right|=2$ or 3 and that $\left|X_{\ell+1}(P)\right| \geq 1$. There are two cases.

Case $1 \quad\left[\left|X_{\ell}(P)\right|=2\right]$ : $\quad$ Lemma 4 and $\ell \geq 1$ imply that $2=\left|X_{\ell}(P)\right|>\left|X_{\ell+1}(P)\right| \cdot(\ell+1) \geq\left|X_{\ell+1}(P)\right| \cdot 2$. Thus, we must have $\left|X_{\ell+1}(P)\right|=0$, a contradiction to $\left|X_{\ell+1}(P)\right| \geq 1$.

Case $2\left[\left|X_{\ell}(P)\right|=3\right]$ : Lemma 3 and $m \leq 4$ imply that $\left|X_{\ell+1}(P)\right|=1$ (i.e., $m=4$ ), and so $F_{p}(P)=X_{\ell+1}(P)$. Let $X_{\ell+1}(P) \equiv\{w\}$. Lemma 4 also implies that $3>1 \cdot(\ell+1)$. Thus, we must have $\ell=1$, and so $n=5$. Let $X_{1}(P) \equiv\{x, y, z\}$. Let 
$j, j^{\prime} \in N$ be such that $t\left(P_{j}\right)=t\left(P_{j^{\prime}}\right)=w$ and let $h, h^{\prime}, h^{\prime \prime} \in N$ be such that $t\left(P_{h}\right)=x$, $t\left(P_{h^{\prime}}\right)=y$, and $t\left(P_{h^{\prime \prime}}\right)=z$. Define $P^{\prime}=\left(P_{i}^{\prime}\right)_{i \in N} \in \mathscr{P}^{N} \quad$ by $\quad P_{j}^{\prime}=\tilde{P}_{2}\left(P_{j} ; y\right)$, $P_{j^{\prime}}^{\prime}=\tilde{P}_{2}\left(P_{j^{\prime}} ; z\right), P_{h}^{\prime}=\tilde{P}_{2}\left(P_{h} ; z\right), P_{h^{\prime}}^{\prime}=\tilde{P}_{2}\left(P_{h^{\prime}} ; x\right)$, and $P_{h^{\prime \prime}}^{\prime}=P_{h^{\prime \prime}}$ (see Fig. 1). Since $P$ and $P^{\prime}$ have CD, we have $f\left(P^{\prime}\right) \in X_{1}\left(P^{\prime}\right)$. Then, we show the following claim.

Claim 1 It holds that $f\left(P^{\prime}\right)=x$.

Proof of Claim 1: Suppose the contrary that $f\left(P^{\prime}\right) \neq x$, i.e., $f\left(P^{\prime}\right) \in\{y, z\}$. Let $f\left(P^{\prime}\right)=y$. Then let $P^{*} \equiv\left(\tilde{P}\left(P_{j}^{\prime} ; y\right), P_{-j}^{\prime}\right) \in \mathscr{P}^{N}$. By weak monotonicity, we have $f\left(P^{*}\right)=y \in X_{2}\left(P^{\prime \prime}\right)$. Note that $P^{\prime}$ and $P^{*}$ have $\mathrm{CD}$. On the other hand, we have $f\left(P^{\prime}\right) \in X_{1}\left(P^{\prime}\right)$ and $f\left(P^{*}\right) \in X_{2}\left(P^{*}\right)$, a contradiction. It is similar for the case of $f\left(P^{\prime}\right)=z$.

We proceed to the proof of Case 2. Define $P^{\prime \prime}=\left(P_{i}^{\prime \prime}\right)_{i \in N} \in \mathscr{P}^{N}$ by $P_{h^{\prime}}^{\prime \prime}=\tilde{P}\left(P_{h^{\prime}}^{\prime} ; x\right)$ and for all $i \neq h^{\prime}, P_{i}^{\prime \prime}=P_{i}^{\prime}$ (see Fig. 1). By Claim 1, weak monotonicity implies that $f\left(P^{\prime \prime}\right)=x$. Next, let $P^{1}=\left(P_{i}^{1}\right)_{i \in N} \in \mathscr{P}^{N}$ be defined as in Fig. 1 . Alternatives on the positions where nothing is displayed are the same as $P^{\prime}$. Then, $P^{\prime}$ and $P^{1}$ have CD. Thus, $f\left(P^{1}\right) \in X_{1}\left(P^{1}\right)$. By the similar argument as the proof of Claim 1, we have $f\left(P^{1}\right)=w$. Let $P^{2}=\left(P_{i}^{2}\right)_{i \in N} \in \mathscr{P}^{N}$ be such that $P_{j}^{2}=\tilde{P}\left(P_{j}^{1} ; w\right)$ and for all $i \neq j, P_{i}^{2}=P_{i}^{1}$ (see Fig. 1). It holds by weak monotonicity that $f\left(P^{2}\right)=w$. However, note that $P^{2}=P^{\prime \prime}$ (see Fig. 1). This is a contradiction since $f\left(P^{2}\right)=w \neq x=f\left(P^{\prime \prime}\right)$.

For the proof of [2] of Theorem 2, we give the following lemma, which generalizes Lemma 3.

Lemma 5 For each $P \in \mathscr{P}^{N}$, each $\ell \geq 1$ and each $\bar{\ell} \geq 2$ with $\ell<\bar{\ell}$, if $f(P) \in X_{\ell}(P)$ and $F_{p}(P)=X_{\bar{\ell}}(P)$, then for all $\ell^{\prime} \in\{\ell+1, \ldots, \bar{\ell}\}, X_{\ell^{\prime}}(P) \neq \emptyset$.

Proof of Lemma 5: Let $P^{\ell}=\left(P_{i}^{\ell}\right)_{i \in N} \in \mathscr{P}^{N}, \ell \geq 1$, and $\bar{\ell} \geq 2$ with $\ell<\bar{\ell}$ be such that $f\left(P^{\ell}\right) \in X_{\ell}\left(P^{\ell}\right)$ and $F_{p}\left(P^{\ell}\right)=X_{\bar{\ell}}\left(P^{\ell}\right) . X_{\bar{\ell}}\left(P^{\ell}\right) \neq \emptyset$ is self-evident. Lemma 3 implies that $X_{\ell+1}\left(P^{\ell}\right) \neq \emptyset$. Thus, the case of $\bar{\ell} \leq \ell+2$ is clear, and so we consider the case of $\bar{\ell}>\ell+2$. In the following, it suffices to show that for all $\ell^{\prime} \in\{\ell+2, \ldots, \bar{\ell}-1\}, X_{\ell^{\prime}}\left(P^{\ell}\right) \neq \emptyset$. The proof of this statement consists of two steps.

Step 1. For all $\ell^{\prime} \in\{\ell+1, \ldots, \bar{\ell}-2\}$, there exists $P^{\ell^{\prime}} \in \mathscr{P}^{N}$ such that

$$
\text { (1) } f\left(P^{\ell^{\prime}}\right) \in X_{\ell^{\prime}}\left(P^{\ell^{\prime}}\right) \text { and (2) for all } \tilde{\ell} \text { with } \tilde{\ell}>\ell^{\prime}, X_{\tilde{\ell}}\left(P^{\ell^{\prime}}\right)=X_{\tilde{\ell}}\left(P^{\ell}\right) \text {. }
$$

We prove by induction on $\ell^{\prime}$. At first, we show the existence of $P^{\ell+1}$ satisfying (1) $f\left(P^{\ell+1}\right) \in X_{\ell+1}\left(P^{\ell+1}\right)$ and (2) for all $\tilde{\ell}$ with $\tilde{\ell}>\ell+1, X_{\tilde{\ell}}\left(P^{\ell+1}\right)=X_{\tilde{\ell}}\left(P^{\ell}\right)$. Note that Lemma 1 implies that $\left|X_{\ell}\left(P^{\ell}\right)\right| \geq 2$. Let $N^{\ell} \subset N$ with $\left|N^{\ell}\right|=\left|X_{\ell}\left(P^{\ell}\right)\right|$ be such that for all $j \in N^{\ell}, t\left(P_{j}^{\ell}\right) \in X_{\ell}\left(P^{\ell}\right)$ and for all $j, j^{\prime} \in N^{\ell}$ with $j \neq j^{\prime}, t\left(P_{j}^{\ell}\right) \neq t\left(P_{j^{\prime}}^{\ell}\right)$. 


$$
\begin{aligned}
& \begin{array}{ccccc}
P_{j}^{\prime} & P_{j^{\prime}}^{\prime} & P_{h}^{\prime} & P_{h^{\prime}}^{\prime} & P_{h^{\prime \prime}}^{\prime} \\
\hline w & w & x & y & z
\end{array} \\
& \begin{array}{ccccc}
P_{j}^{\prime \prime} & P_{j^{\prime}}^{\prime \prime} & P_{h}^{\prime \prime} & P_{h^{\prime}}^{\prime \prime} & P_{h^{\prime \prime}}^{\prime \prime} \\
\hline w & w & x & x & z
\end{array} \\
& P^{\prime}= \\
& P^{\prime \prime}=\begin{array}{llll} 
& & & \\
& z & y
\end{array} \\
& \begin{array}{rrrrr}
P_{j}^{1} & P_{j^{\prime}}^{1} & P_{h}^{1} & P_{h^{\prime}}^{1} & P_{h^{\prime \prime}}^{1} \\
\hline y & w & x & x & z
\end{array} \\
& P^{1}= \\
& \begin{array}{ccccc}
P_{j}^{2} & P_{j^{\prime}}^{2} & P_{h}^{2} & P_{h^{\prime}}^{2} & P_{h^{\prime \prime}}^{2} \\
\hline w & w & x & x & z
\end{array} \\
& P^{2}=
\end{aligned}
$$

Fig. 1 Profiles $P^{\prime}, P^{\prime \prime}, P^{1}$, and $P^{2}$ in Case 2 of Proof of [1] of Theorem 2

Then, we can construct a bijection $g_{\ell}: N^{\ell} \rightarrow X_{\ell}\left(P^{\ell}\right)$ such that for all $j \in N^{\ell}$, $g_{\ell}(j) \neq t\left(P_{j}^{\ell}\right)$. Let $P^{\ell *}=\left(P_{i}^{\ell *}\right)_{i \in N} \in \mathscr{P}^{N}$ be defined by

$$
\text { for all } j \in N^{\ell}, P_{j}^{\ell *}=\tilde{P}_{2}\left(P_{j}^{\ell} ; g_{\ell}(j)\right) \text { and for all } j \notin N^{\ell}, P_{j}^{\ell *}=P_{j}^{\ell} .
$$

Then since for all $j \in N, t\left(P_{j}^{\ell}\right)=t\left(P_{j}^{\ell *}\right), P^{\ell}$ and $P^{\ell *}$ have CD. Thus, by ETCD, $f\left(P^{\ell *}\right) \equiv x^{\ell *} \in X_{\ell}\left(P^{\ell *}\right)=X_{\ell}\left(P^{\ell}\right)$. By the definition of $P^{\ell *}$, there is $i^{\ell *} \in N^{\ell}$ such that $t_{2}\left(P_{i \ell^{\ell *}}^{\ell *}\right)=x^{\ell *}$. Let $P^{\ell+1} \equiv\left(\tilde{P}\left(P_{i \ell^{\ell *}}^{\ell *} x^{\ell *}\right), P_{-i \ell^{\ell *}}^{\ell *}\right) \in \mathscr{P}^{N}$. Weak monotonicity implies that $f\left(P^{\ell+1}\right)=x^{\ell *}$. Note that $x^{\ell^{\ell *}} \in X_{\ell+1}\left(P^{\ell+1}\right)$. Furthermore, by the definition of $P^{\ell+1}$, for all $\tilde{\ell}>\ell+1, X_{\tilde{\ell}}\left(P^{\ell+1}\right)=X_{\tilde{\ell}}\left(P^{\ell}\right)$.

Next, given $\ell^{\prime} \in\{\ell+1, \ldots, \bar{\ell}-3\},{ }^{30}$ suppose that there exists $P^{\ell^{\prime}} \in \mathscr{P}^{N}$ such that (1) $f\left(P^{\ell^{\prime}}\right) \in X_{\ell^{\prime}}\left(P^{\ell^{\prime}}\right)$ and (2) for all $\tilde{\ell}$ with $\tilde{\ell}>\ell^{\prime}, X_{\tilde{\ell}}\left(P^{\ell^{\prime}}\right)=X_{\tilde{\ell}}\left(P^{\ell}\right)$. We then show that there exists $P^{\ell^{\prime}+1} \in \mathscr{P}^{N}$ such that $(1)^{\prime} f\left(P^{\ell^{\prime}+1}\right) \in X_{\ell^{\prime}+1}\left(P^{\ell^{\prime}+1}\right)$ and $(2)^{\prime}$ for all $\tilde{\ell}$ with $\tilde{\ell}>\ell^{\prime}+1, X_{\tilde{\ell}}\left(P^{\ell^{\prime}+1}\right)=X_{\tilde{\ell}}\left(P^{\ell}\right)$. By (2), $F_{p}\left(P^{\ell^{\prime}}\right)=X_{\bar{\ell}}\left(P^{\ell^{\prime}}\right)$, and so by (1), $f\left(P^{\ell^{\prime}}\right) \notin F_{p}\left(P^{\ell^{\prime}}\right)$. Thus, Lemma 1 implies that $\left|X_{\ell^{\prime}}\left(P^{\ell^{\prime}}\right)\right| \geq 2$. Let $N^{\ell^{\prime}} \subset N$ with $\left|N^{\ell^{\prime}}\right|=\left|X_{\ell^{\prime}}\left(P^{\ell^{\prime}}\right)\right|$ be such that for all $j \in N^{\ell^{\prime}}, t\left(P_{j}^{\ell^{\prime}}\right) \in X_{\ell^{\prime}}\left(P^{\ell^{\prime}}\right)$ and for all $j, j^{\prime} \in N^{\ell^{\prime}}$ with $j \neq j^{\prime}, t\left(P_{j}^{\ell^{\prime}}\right) \neq t\left(P_{j^{\prime}}^{\ell^{\prime}}\right)$. Then, we can construct a bijection $g_{\ell^{\prime}}: N^{\ell^{\prime}} \rightarrow X_{\ell^{\prime}}\left(P^{\ell^{\prime}}\right)$ such that for all $j \in N^{\ell^{\prime}}, g_{\ell^{\prime}}(j) \neq t\left(P_{j}^{\ell^{\prime}}\right)$. Let $P^{\ell^{\prime *}}=\left(P_{i}^{\ell^{\prime} *}\right)_{i \in N} \in \mathscr{P}^{N}$ be defined by

for all $j \in N^{\ell^{\prime}}, P_{j}^{\ell^{\prime} *}=\tilde{P}_{2}\left(P_{j}^{\ell^{\prime}} ; g_{\ell^{\prime}}(j)\right)$ andforallj $\notin N^{\ell^{\prime}}, P_{j}^{\ell^{\prime} *}=P_{j}^{\ell^{\prime}}$.

Then since for all $j \in N, t\left(P_{j}^{\ell^{\prime}}\right)=t\left(P_{j}^{\ell^{\prime} *}\right), P^{\ell^{\prime}}$ and $P^{\ell^{\prime} *}$ have CD. Thus, by ETCD, $f\left(P^{\ell^{\prime} *}\right) \equiv x^{\ell^{\prime} *} \in X_{\ell^{\prime}}\left(P^{\ell^{\prime} *}\right)=X_{\ell^{\prime}}\left(P^{\ell^{\prime}}\right)$. By the definition of $P^{\ell^{\prime} *}$, there is $i^{\ell^{\prime} *} \in N^{\ell^{\prime}}$ such that $t_{2}\left(P_{i^{\ell^{\prime} *}}^{\ell^{\prime} *}\right)=x^{\ell^{\prime} *}$. Let $P^{\ell^{\prime}+1} \equiv\left(\tilde{P}\left(P_{i^{\ell^{\prime} *}}^{\ell^{\prime} *} ; x^{\ell^{\prime} *}\right), P_{-i^{\ell^{\prime} *}}^{\ell^{\prime} *}\right) \in \mathscr{P}^{N}$. Weak monotonicity implies that $f\left(P^{\ell^{\prime}+1}\right)=x^{\ell^{\prime} *}$. Note that $x^{\ell^{\prime} *} \in X_{\ell^{\prime}+1}\left(P^{\ell^{\prime}+1}\right)$. Furthermore, by the

\footnotetext{
${ }^{30}$ In this part, it is implicitly assumed that $\bar{\ell}-3 \geq \ell+1$ (i.e., $\bar{\ell}>\ell+3$ ). For the proof of the case of $\bar{\ell}=\ell+3$, we have already proved it in the first part of the induction, i.e., the proof of the existence of $P^{\ell+1}$ (Recall that Step 1 shows the existence of $(\bar{\ell}-2-\ell)$-profile(s)).
} 
definition of $P^{\ell^{\prime}+1}$, for all $\tilde{\ell}>\ell^{\prime}+1, X_{\tilde{\ell}}\left(P^{\ell^{\prime}+1}\right)=X_{\tilde{\ell}}\left(P^{\ell^{\prime}}\right)=X_{\tilde{\ell}}\left(P^{\ell}\right)$. The last equality follows from (2). Thus, $P^{\ell^{\prime}+1}$ satisfies (1)' and (2)'.

Step 2. For all $\ell^{\prime} \in\{\ell+2, \ldots, \bar{\ell}-1\}, X_{\ell^{\prime}}\left(P^{\ell}\right) \neq \emptyset$.

Given $\ell^{\prime} \in\{\ell+2, \ldots, \bar{\ell}-1\}$. By Step 1 , there exists $P^{\ell^{\prime}-1} \in \mathscr{P}^{N}$ such that $f\left(P^{\ell^{\prime}-1}\right) \in X_{\ell^{\prime}-1}\left(P^{\ell^{\prime}-1}\right)$ and for all $\tilde{\ell}$ with $\tilde{\ell}>\ell^{\prime}-1, \quad X_{\tilde{\ell}}\left(P^{\ell^{\prime}-1}\right)=X_{\tilde{\ell}}\left(P^{\ell}\right)$. Recall that these properties of $P^{\ell^{\prime}-1}$ imply that $F_{p}\left(P^{\ell^{\prime}-1}\right)=X_{\bar{\ell}}\left(P^{\ell^{\prime}-1}\right)$ and $f\left(P^{\ell^{\prime}-1}\right) \notin F_{p}\left(P^{\ell^{\prime}-1}\right)$. Thus, by Lemma 3 with $f\left(P^{\ell^{\prime}-1}\right) \in X_{\ell^{\prime}-1}\left(P^{\ell^{\prime}-1}\right)$, $X_{\ell^{\prime}}\left(P^{\ell^{\prime}-1}\right) \neq \emptyset$, which in turn implies that $X_{\ell^{\prime}}\left(P^{\ell}\right) \neq \emptyset$.

Proof of [2] of Theorem 2: Let $m \geq 3$ be given. ${ }^{31}$ Let

$$
\bar{n} \equiv \max \left\{\frac{(2 m-4)^{3} m-4 m-4}{3}, \frac{(2 m-4)^{2} m}{3}\right\} .
$$

Let $n$ be an integer with $n \geq \bar{n}$. By $m \geq 3$, it holds that $n \geq \bar{n}>m$. That is, $(m, n)$ satisfies the assumption of Lemma 2. Suppose the contrary that $f(P) \notin F_{p}(P)$ for some $P \in \mathscr{P}^{N}$. Let $\bar{\ell}$ be such that $X_{\bar{\ell}}(P)=F_{p}(P)$. By Lemma 2, we have $f(P) \in X_{\ell}(P)$ for some $\ell \in\{1, \ldots, \bar{\ell}-1\}$. At first, we show the following claim.

Claim 2 It holds that $\bar{\ell}<2 m-4$.

Proof of Claim 2: By $\ell \geq 1$ and Lemmas 3 and 4, we have $\left|X_{\ell}(P)\right|>\left|X_{\ell+1}(P)\right| \cdot(\ell$ $+1) \geq 2$. Note that $m \geq\left|X_{\ell}(P)\right|+\left|X_{\ell+1}(P)\right|+\cdots+\left|X_{\bar{\ell}}(P)\right| \geq 3+1+\cdots+1=3$ $+\bar{\ell}-\ell$. The first inequality follows from the definition of $m$ and the second one follows from Lemma 5. Thus, we have $\bar{\ell} \leq \ell+m-3$. Furthermore, by Lemmas 3 and 4 , we also have $\left|X_{\ell}(P)\right| \geq\left|X_{\ell}(P)\right| /\left|X_{\ell+1}(P)\right|>\ell+1$. Thus, by $\left|X_{\ell}(P)\right|<m$, we obtain that $\ell<\left|X_{\ell}(P)\right|-1<m-1$. Therefore, it holds that $\bar{\ell} \leq \ell+m-3<m-1+m-3=2 m-4$.

We proceed to the proof of [2] of Theorem 2. By the definitions of $m$ and $n$, the following two identities hold.

$$
\begin{aligned}
& \left|X_{0}(P)\right|+\left|X_{1}(P)\right|+\cdots+\left|X_{\bar{\ell}}(P)\right|=m \text { and } \\
& \quad 1 \cdot\left|X_{1}(P)\right|+2 \cdot\left|X_{2}(P)\right|+\cdots+\bar{\ell} \cdot\left|X_{\bar{\ell}}(P)\right|=n .
\end{aligned}
$$

There are two cases.

Case $1[\bar{\ell}>\ell+1]$ : Solving $(* *)$ for $\left|X_{\ell}(P)\right|$ and $\left|X_{\ell+1}(P)\right|$, we obtain that

\footnotetext{
$\overline{31}$ As mentioned at the beginning of this appendix, the proof of [2] can apply to all $m \geq 3$.
} 


$$
\begin{aligned}
& \left|X_{\ell}(P)\right|=(\ell+1) m-n-\sum_{\ell^{\prime}=0}^{\ell-1}\left(\ell+1-\ell^{\prime}\right)\left|X_{\ell^{\prime}}(P)\right|+\sum_{\ell^{\prime}=\ell+2}^{\bar{\ell}}\left(\ell^{\prime}-\ell-1\right)\left|X_{\ell^{\prime}}(P)\right| \text { and } \\
& \left|X_{\ell+1}(P)\right|=n-\ell m+\sum_{\ell^{\prime}=0}^{\ell-1}\left(\ell-\ell^{\prime}\right)\left|X_{\ell^{\prime}}(P)\right|-\sum_{\ell^{\prime}=\ell+2}^{\bar{\ell}}\left(\ell^{\prime}-\ell\right)\left|X_{\ell^{\prime}}(P)\right| .
\end{aligned}
$$

Then, we have

$$
\begin{aligned}
& (\ell+1) \cdot\left|X_{\ell+1}(P)\right|-\left|X_{\ell}(P)\right| \\
& =(\ell+2) n-(\ell+1)^{2} m+(\ell+1)\left\{\sum_{\ell^{\prime}=0}^{\ell-1}\left(\ell-\ell^{\prime}\right)\left|X_{\ell^{\prime}}(P)\right|\right. \\
& \left.-\sum_{\ell^{\prime}=\ell+2}^{\bar{\ell}}\left(\ell^{\prime}-\ell\right)\left|X_{\ell^{\prime}}(P)\right|\right\}+\sum_{\ell^{\prime}=0}^{\ell-1}\left(\ell+1-\ell^{\prime}\right)\left|X_{\ell^{\prime}}(P)\right| \\
& -\sum_{\ell^{\prime}=\ell+2}^{\bar{\ell}}\left(\ell^{\prime}-\ell-1\right)\left|X_{\ell^{\prime}}(P)\right| \\
& \geq(\ell+2) n-(\ell+1)^{2} m-(\ell+1) \sum_{\ell^{\prime}=\ell+2}^{\bar{\ell}}\left(\ell^{\prime}-\ell\right)\left|X_{\ell^{\prime}}(P)\right|-\sum_{\ell^{\prime}=\ell+2}^{\bar{\ell}}\left(\ell^{\prime}-\ell-1\right)\left|X_{\ell^{\prime}}(P)\right| \\
& \geq 3 n-(\ell+1)^{2} m-(\ell+1) \sum_{\ell^{\prime}=\ell+2}^{\bar{\ell}}\left(\ell^{\prime}-\ell\right)\left|X_{\ell^{\prime}}(P)\right| \\
& -\sum_{\ell^{\prime}=\ell+2}^{\bar{\ell}}\left(\ell^{\prime}-\ell-1\right)\left|X_{\ell^{\prime}}(P)\right| \quad(\text { by } \ell \geq 1) \\
& =3 n-(\ell+1)^{2} m-(\ell+1) \sum_{\ell^{\prime}=\ell+2}^{\bar{\ell}} \ell^{\prime}\left|X_{\ell^{\prime}}(P)\right|+(\ell+1) \ell \sum_{\ell^{\prime}=\ell+2}^{\bar{\ell}}\left|X_{\ell^{\prime}}(P)\right| \\
& -\sum_{\ell^{\prime}=\ell+2}^{\bar{\ell}} \ell^{\prime}\left|X_{\ell^{\prime}}(P)\right|+(\ell+1) \sum_{\ell^{\prime}=\ell+2}^{\bar{\ell}}\left|X_{\ell^{\prime}}(P)\right| \\
& =3 n-(\ell+1)^{2} m-(\ell+2) \sum_{\ell^{\prime}=\ell+2}^{\bar{\ell}} \ell^{\prime}\left|X_{\ell^{\prime}}(P)\right|+(\ell+1)^{2} \sum_{\ell^{\prime}=\ell+2}^{\bar{\ell}}\left|X_{\ell^{\prime}}(P)\right| \\
& \geq 3 n-(\ell+1)^{2} m-(\ell+2) \sum_{\ell^{\prime}=\ell+2}^{\bar{\ell}} \ell^{\prime}\left|X_{\ell^{\prime}}(P)\right|+(\ell+1)^{2} \sum_{\ell^{\prime}=\ell+2}^{\bar{\ell}} 1 \quad \text { (by Lemma 5) }
\end{aligned}
$$




$$
\begin{aligned}
= & 3 n-(\ell+1)^{2} m-(\ell+2) \sum_{\ell^{\prime}=\ell+2}^{\bar{\ell}} \ell^{\prime}\left|X_{\ell^{\prime}}(P)\right|+(\ell+1)^{2}(\bar{\ell}-\ell-1) \\
> & 3 n-\bar{\ell}^{2} m-(\bar{\ell}+1) \sum_{\ell^{\prime}=\ell+2}^{\bar{\ell}} \ell^{\prime}\left|X_{\ell^{\prime}}(P)\right|+4 \quad(\text { by } \bar{\ell}>\ell+1 \text { and } \ell \geq 1) \\
> & 3 n-\bar{\ell}^{2} m-(\bar{\ell}+1) \sum_{\ell^{\prime}=\ell+2}^{\bar{\ell}} \bar{\ell} m+4 \\
& \left(\text { by for all } \ell^{\prime} \in\{\ell+2, \ldots, \bar{\ell}\}, \ell^{\prime} \leq \bar{\ell} \text { and }\left|X_{\ell^{\prime}}(P)\right|<m\right) \\
= & 3 n-\bar{\ell}^{2} m-(\bar{\ell}+1)(\bar{\ell}-\ell-1) \bar{\ell} m+4 \\
\geq & 3 n-\bar{\ell}^{2} m+(\bar{\ell}+1)(2-\bar{\ell}) \bar{\ell} m+4 \quad(\text { by } \ell \geq 1) \\
= & 3 n-\bar{\ell}\left(\bar{\ell}^{2}-2\right) m+4 \quad(\text { by } \bar{\ell}>\ell+1 \geq 2) \\
> & 3 n-\bar{\ell}^{3} m+4 m+4 \quad(\text { by Claim } 2) \\
> & 3 n-(2 m-4)^{3} m+4 m+4 \quad \\
\geq & 0 \quad\left(\text { by } n \geq \bar{n} \geq\left\{(2 m-4)^{3} m-4 m-4\right\} / 3\right) .
\end{aligned}
$$

This is a contradiction to Lemma 4.

Case $2[\bar{\ell}=\ell+1]$ : Solving $(* *)$ for $\left|X_{\ell}(P)\right|$ and $\left|X_{\ell+1}(P)\right|$, we obtain that

$$
\begin{aligned}
& \left|X_{\ell}(P)\right|=(\ell+1) m-n-\sum_{\ell^{\prime}=0}^{\ell-1}\left(\ell+1-\ell^{\prime}\right)\left|X_{\ell^{\prime}}(P)\right| \text { and } \\
& \left|X_{\ell+1}(P)\right|=n-\ell m+\sum_{\ell^{\prime}=0}^{\ell-1}\left(\ell-\ell^{\prime}\right)\left|X_{\ell^{\prime}}(P)\right| .
\end{aligned}
$$

Then, we have

$$
\begin{aligned}
(\ell & +1) \cdot\left|X_{\ell+1}(P)\right|-\left|X_{\ell}(P)\right| \\
& =(\ell+2) n-(\ell+1)^{2} m+(\ell+1) \sum_{\ell^{\prime}=0}^{\ell-1}\left(\ell-\ell^{\prime}\right)\left|X_{\ell^{\prime}}(P)\right|+\sum_{\ell^{\prime}=0}^{\ell-1}\left(\ell+1-\ell^{\prime}\right)\left|X_{\ell^{\prime}}(P)\right| \\
& \geq(\ell+2) n-(\ell+1)^{2} m \\
& \geq 3 n-\bar{\ell}^{2} m \quad(\text { by } \ell \geq 1 \text { and } \bar{\ell}=\ell+1) \\
& >3 n-(2 m-4)^{2} m \quad(\text { by Claim } 2) \\
& \geq 0 \quad\left(\text { by } n \geq \bar{n} \geq(2 m-4)^{2} m / 3\right) .
\end{aligned}
$$

This is a contradiction to Lemma 4.

Acknowledgements The author is grateful to the anonymous reviewers for helpful comments and suggestions on an earlier version of the paper. 
Funding The author did not receive support from any organization for the submitted work.

Availability of data and material Not applicable.

\section{Declarations}

Conflict of interest The author has no conflicts of interest to declare that are relevant to the content of this article.

Code availability Not applicable.

Open Access This article is licensed under a Creative Commons Attribution 4.0 International License, which permits use, sharing, adaptation, distribution and reproduction in any medium or format, as long as you give appropriate credit to the original author(s) and the source, provide a link to the Creative Commons licence, and indicate if changes were made. The images or other third party material in this article are included in the article's Creative Commons licence, unless indicated otherwise in a credit line to the material. If material is not included in the article's Creative Commons licence and your intended use is not permitted by statutory regulation or exceeds the permitted use, you will need to obtain permission directly from the copyright holder. To view a copy of this licence, visit http://creativecommons.org/licen ses/by/4.0/.

\section{References}

Baharad E, Nitzan S (2005) The inverse plurality rule-an axiomatization. Soc Choice Welf 25:173-178

Bubboloni D, Gori M (2014) Anonymous and neutral majority rules. Soc Choice Welf 43:377-401

Bubboloni D, Gori M (2021) Breaking ties in collective decision-making. Decis Econ Financ 44:411-457

Campbell DE, Kelly JS (2013) Anonymity, monotonicity, and limited neutrality: selecting a single alternative from a binary agenda. Econ Lett 118:10-12

Campbell DE, Kelly JS (2015) The finer structure of resolute, neutral, and anonymous social choice correspondences. Econ Lett 132:109-111

Campbell DE, Kelly JS (2016) A strategy-proofness characterization of plurality rule. J Public Econ Theory 18:610-623

Ching S (1996) A simple characterization of plurality rule. J Econ Theory 71:298-302

Doğan O, Giritligil AE (2015) Anonymous and neutral social choice: existence results on resoluteness. Murat Sertel Center for Advanced Economic Studies Working Paper Series No: 2015-01

Fishburn PC (1977) Condorcet social choice functions. SIAM J Appl Math 33:469-489

Fishburn PC, Gehrlein WV (1977) Collective rationality versus distribution of power for binary social choice functions. J Econ Theory 15:72-91

Gibbard A (1973) Manipulation of voting schemes: a general result. Econometrica 41:587-602

Jeong H, Ju BG (2017) Resolute majority rules. Theory Decis 82:31-39

Ju BG (2015) An efficiency characterization of plurality social choice on simple preference domains. Econ Theory 26:115-128

Kelly JS, Qi S (2016) Characterizing plurality rule on a fixed population. Econ Lett 146:39-41

Laffond G, Lainé J, Laslier J-F (1996) Composition-consistent tournament solutions and social choice functions. Soc Choice Welf 13:75-93

Laslier J-F (1996) Rank-based choice correspondences. Econ Lett 52:279-286

Laslier J-F (2000) Aggregation of preferences with a variable set of alternatives. Soc Choice Welf 17:269-282

May KO (1952) A set of independent necessary and sufficient conditions for simple majority decision. Econometrica 20:680-684

Moulin H (1979) Dominance solvable voting schemes. Econometrica 47:1337-1351

Moulin H (1980) Implementing efficient, anonymous and neutral social choice functions. J Math Econ 7:249-269 
Moulin H (1983) The strategy of social choice. North-Holland, Amsterdam

Moulin H (1988) Condorcet's principle implies the no show paradox. J Econ Theory 45:53-64

Nitzan S, Paroush J (1981) The characterization of decisive weighted majority rules. Econ Lett 7:119-124

Ozkes AI, Sanver MR (2021) Anonymous, neutral, and resolute social choice revisited. Soc Choice Welf 57:97-113

Öztürk ZE (2020) Consistency of scoring rules: a reinvestigation of composition-consistency. Int J Game Theory 49:801-831

Pazner EA, Wesley E (1978) Cheatproofness properties of the plurality rule in large societies. Rev Econ Stud 45:85-91

Peleg B (1978) Consistent voting systems. Econometrica 46:153-161

Richelson JT (1978) A characterization result for the plurality rule. J Econ Theory 19:548-550

Sanver MR (2009) Strategy-proofness of the plurality rule over restricted domains. Econ Theory 39:461-471

Satterthwaite MA (1975) Strategy-proofness and Arrow's conditions: existence and correspondence theorems for voting procedures and social welfare functions. J Econ Theory 10:187-217

Schulze M (2011) A new monotonic, clone-independent, reversal symmetric, and Condorcet-consistent single-winner election method. Soc Choice Welf 36:267-303

Sekiguchi Y (2012) A characterization of the plurality rule. Econ Lett 116:330-332

Tideman TN (1987) Independence of clones as a criterion for voting rules. Soc Choice Welf 4:185-206

Yeh C-H (2006) Reduction-consistency in collective choice problems. J Math Econ 42:637-652

Yeh C-H (2008) An efficiency characterization of plurality rule in collective choice problems. Econ Theory $34: 575-583$

Young HP (1974) An axiomatization of Borda's rule. J Econ Theory 9:43-52

Young HP (1975) Social choice scoring functions. SIAM J Appl Math 28:824-838

Zavist TM, Tideman TN (1989) Complete independence of clones in the ranked pairs rule. Soc Choice Welf 6:167-173

Publisher's Note Springer Nature remains neutral with regard to jurisdictional claims in published maps and institutional affiliations. 